\title{
On the Equivalence of Schemes for Simulating Bilayers at Constant Surface Tension
}

\author{
Jocelyn M. Rodgers ${ }^{* \dagger}$ and Berend Smit*,\$,\$,\| \\ ${ }^{\dagger}$ Physical Biosciences Division, Lawrence Berkeley National Laboratory, Berkeley, California 94720, United States \\ ${ }^{\ddagger}$ Department of Chemical and Biomolecular Engineering, University of California, Berkeley, 101B Gilman Hall, Berkeley, California \\ 94720-1462, United States \\ ${ }^{\S}$ Materials Science Division, Lawrence Berkeley National Laboratory, Berkeley, California 94720, United States \\ "Department of Chemistry, University of California, Berkeley, 101B Gilman Hall, Berkeley, California 94720-1462, United States
}

\section{Supporting Information}

ABSTRACT: Lipid bilayers are simulated using flexible simulation cells in order to allow for relaxations in area per lipid as bilayer content and temperature are varied. We develop a suite of Monte Carlo (MC) moves designed to generate constant surface tension $\gamma$ and constant pressure $P$ and find that the NPT partition function proposed by Attard [J. Chem. Phys. 1995, 103, 9884-9885] leads to an $N P \gamma T$ partition function with a form invariant to choice of independent shape variables. We then compare this suite of MC moves to NP $\gamma T$ MC moves previously employed in our group as well as a pair of MC moves designed to replicate the $N P_{\|} P_{\perp} T$ "ensemble" often used in molecular dynamics simulations to yield zero surface tension and constant pressure. A detailed analysis of shape fluctuations in a small bilayer system reveals that the two latter MC move sets are different from one another as well as from our new suite of MC moves, as justified by careful analysis of the partition functions. However, the study of a larger bilayer system reveals that, for practical purposes for this system, all six MC move sets are comparable to one another.

\section{INTRODUCTION}

Molecular simulation of lipid bilayers has yielded insight into a wide range of biophysical membrane processes. ${ }^{1-4}$ Experimental systems are often lipid vesicles or cells with a large (macroscopic) number of lipids contained within the closed lipid bilayer envelope. Simulating such large systems is well beyond the capabilities of molecular simulations, whether atomic or coarse-grained. Therefore, simulations are instead conducted using a tetragonal simulation box, as shown in Figure 1.

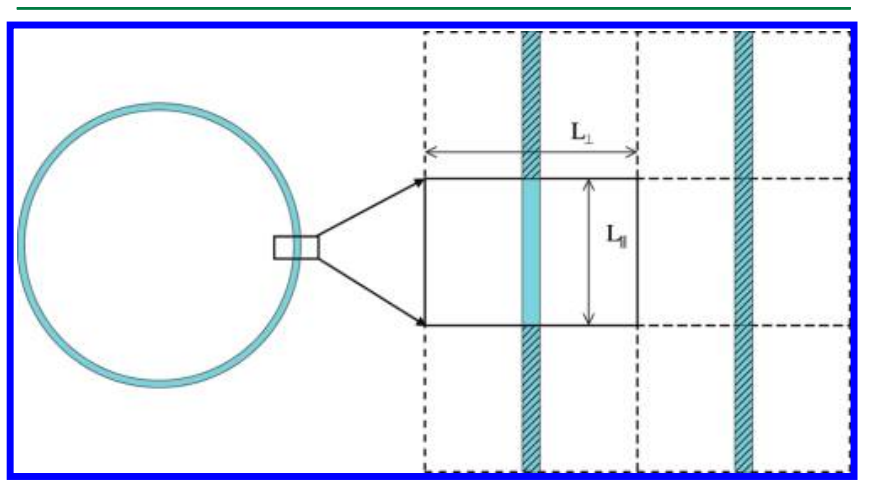

Figure 1. Schematic of the mapping of a portion of an experimental lipid vesicle to a simulation box with side lengths $L_{\|}$and $L_{\perp}$ as well as periodic boundary conditions. For this simulation box geometry, $A \equiv L_{\|}{ }^{2}$ and $V \equiv A \cdot L_{\perp}$. The main image is shown with solid lines and pure cyan for the bilayer. Several neighboring periodic images are displayed with dashed lines and shaded cyan. The white regions correspond to the solvent. Just as the overall surface area of a vesicle composed of a fixed number of lipids may vary, so may the surface area of the lipid molecules in the simulation box adjust in the $N P \gamma T$ ensemble.
Experimentally, the area per lipid in a lipid bilayer varies as a function of both temperature and bilayer composition. For example, the addition of ethanol to a dimyristoyl phosphatidylcholine (DMPC) bilayer induces the formation of an interdigitated phase with nearly double the bilayer area. ${ }^{5}$ While vesicles expand or contract to allow for such modifications in areal density, a standard canonical ensemble simulation of the tetragonal simulation box does not allow for the necessary box shape changes.

In the literature, different schemes have been proposed to allow for changes in box shape during simulation. Zhang et al. developed a tension-stat for molecular dynamics to maintain a constant surface tension $\gamma$ in the plane of the bilayer as well as a constant pressure $P$ normal to the bilayer, an approach which is implemented in CHARMM. ${ }^{6}$ This tension-stat is not available in all molecular dynamics packages, and therefore, many simulators use a barostat with anisotropic coupling, applying the same pressure tangential and normal to the bilayer $\left(P_{\|}=P_{\perp}\right)$ while decoupling shape changes in the tangential and normal direction, with the goal of simulating a tensionless bilayer. ${ }^{7}$ Within our group, as done for molecular dynamics by Zhang et al., ${ }^{6}$ we have developed Monte Carlo moves to simulate a constant surface tension and a constant pressure. ${ }^{3,8,9}$

As these schemes use different approaches, one may reasonably ask whether they are equivalent. Results using these different approaches on different lipid bilayer models often give qualitatively and sometimes quantitatively similar results for bilayers of the same phase. ${ }^{3,10}$ In this work, we aim to make a

Received: October 11, 2011

Published: January 31, 2012 
more formal comparison of the approaches for simulating a tensionless bilayer. We show how different Monte Carlo schemes can be obtained from the same partition function by transforming among different sets of integration variables. As described in section 2, we formulate this study by carefully developing an $N P \gamma T$ partition function, allowing for a set surface tension and pressure. In this effort, we build on the representation of the NPT partition function proposed by Attard $^{11}$ rather than the standard NPT partition function employed in most simulations. ${ }^{12}$ In section 3 , we derive the set of Monte Carlo moves tested for generating the NP $\mathrm{T} T$ ensemble. Subsequently, we present our simple model system-a lipid bilayer in water-in section 4 . We present and discuss our numerical results in section 5 and further explore the reasons for inequivalencies between the old $N P \gamma T$ and $N P_{\|} P_{\perp} T$ schemes in section 6 .

\section{THE NP $\gamma T$ ENSEMBLE}

2.1. NPyT Thermodynamics. In analogy with using constant pressure simulations to allow for density changes in bulk fluids, variation in lateral lipid packing may be simulated by applying a constant pressure and surface tension. Such simulations correspond to modeling the free energy $\mathcal{F}$ governed by the Legendre transform

$$
\begin{aligned}
\mathcal{F} & \left(N_{\text {lip }}, N_{\mathrm{w}}, P, \gamma, T\right) \\
& =\min _{V, A}\left\{F\left(N_{\text {lip }}, N_{\mathrm{w}}, V, A, T\right)+P V-\gamma A\right\}
\end{aligned}
$$

with the differential

$$
d \mathcal{F}=-T d S+V d P-\gamma d A+\mu_{\text {lip }} d N_{\text {lip }}+\mu_{\mathrm{w}} d N_{\mathrm{w}}
$$

An assumption inherent to writing eq 1 is that we are simulating a system where specifying $P, \gamma$, and $T$ simultaneously is meaningful. In order for this to be true, a surface phase must exist. For example, a simple oil-water system has two components, but the interfacial tension between the oil and water phases cannot be chosen independently from the bulk pressure and temperature. For a water-lipid system, if a bilayer is formed, the system can, for a given pressure and temperature, adjust the surface tension of the membrane by changing its number of lipids per area. More formally, in the case of a system with two components, one bulk phase, and one surface phase, we have a total of three degrees of freedom, ${ }^{13,14}$ allowing the specification of $P, \gamma$, and T. Also note that from here forward, we shall simply write $N$ to indicate both $N_{\text {w }}$ and $N_{\text {lip }}$.

The first step in simulating a system defined by the variables $N, P, \gamma$, and $T$ is constructing the partition function $Z_{N P \gamma T}$ for the ensemble. The partition function is related to the free energy as $-\beta \mathcal{F}(N, P, \gamma, T)=\ln Z_{N P \gamma T}$. The simulations then sample from the distribution defined by that $Z_{N P \gamma T}$.

2.2. Previous NPYT Approaches. In many studies, the lipid bilayer is simulated in a tensionless state, as this has been argued to correspond to the free energy minimum of the full vesicular system, $3,15,16$ though early work with all-atom simulations indicated that positive tension may be required for experimental agreement. ${ }^{17}$ For $\gamma=0$ systems, two different choices for simulation are often applied:

- applying a pressure $P_{\perp}$ normal to the bilayer and zero surface tension $\gamma$ in the plane of the bilayer $(N P \gamma T)^{6,8,9}$ or

- applying the same pressure normal to the bilayer and in the plane of the bilayer so that $P_{\perp}=P_{\|}$yet the shape is decoupled in the normal and lateral directions $\left(N P_{\|} P_{\perp} T\right)^{7}$

Both approaches allow for the needed shape changes as lipid bilayers vary temperature and move into different phases. However, the equivalence of the $N P \gamma T$ scheme and the $N P_{\|} P_{\perp} T$ scheme for simulating tensionless bilayers is not proven. When considering the integrand of the partition function associated with the NPrT ensemble,

$$
\exp \left(-\beta P V+\beta \gamma A-\beta U\left(\mathbf{r}^{N}\right)\right)
$$

this equivalence seems compelling, as setting $\gamma=0$ results in an integrand similar to that of the NPT ensemble. In ref 6, Zhang et al. show that the equations of evolution for their barostat/ tension-stat derived to maintain constant pressure and surface tension in molecular dynamics are equivalent to applying the same pressure in lateral and normal directions when $\gamma=0,{ }^{6}$ but we have not found a general demonstration of the equivalence of these two ensembles.

2.3. Formulating the NPyT Partition Function. We now

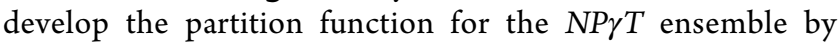
building on that for the NPT ensemble. The traditional partition function employed for simulation of the NPT ensemble ${ }^{12}$ is

$$
\begin{aligned}
Z_{N P T} & =\frac{1}{V_{0}} \int_{0}^{\infty} \mathrm{d} V \mathrm{e}^{-\beta P V} \frac{V^{N}}{N ! \Lambda_{T}^{3 N}} \int \mathrm{d} s^{N} \mathrm{e}^{-\beta U_{N}\left(s^{N} ; V\right)} \\
& =\frac{1}{V_{0}} \int_{0}^{\infty} \mathrm{d} V \mathrm{e}^{-\beta P V} Z(N, V, T)
\end{aligned}
$$

In its basic essence, the above partition function is the NVT partition function integrated over all possible volumes and weighted by the Boltzmann factor composed of the volume and its conjugate variable, pressure. The presence of the prefactor $1 / V_{0}$ has no thermodynamic significance but is required by dimensional analysis. The partition function $Z_{\mathrm{NPT}}$ should be a pure number, and dividing by an arbitrary length scale $V_{0}$ cancels the unit of length resulting from the integration over $V$. This form of the partition function is the one most commonly used for simulating in the NPT ensemble. However, this form is not entirely consistent with either the NVT or $\mu V T$ ensembles. As one example, this formulation leads to an ideal gas law of $P V=(N+1) k_{\mathrm{B}} T$. The error in density is $1 / V$, and in the thermodynamic limit, where $N \rightarrow \infty$ and $V \rightarrow \infty$ while $N / V$ is constant, this difference is vanishingly small. Yet for small systems, as are some simulations, this error for even the ideal gas is meaningful.

In the following, we present the formalism of Attard ${ }^{11}$ to address the deficiency of the standard NPT ensemble. This approach is straightforwardly extended to the NP $\gamma T$ ensemble and seems most appropriate for simulation systems employing periodic boundary conditions (PBC). However, other authors have made compelling arguments for the origin of the difficulties of the NPT ensemble when embedded in a generalized pressure bath. ${ }^{18-20}$ In such cases, the fundamental difficulty with the partition function as expressed in eq 3 lies in overcounting the number of states available at a given volume $V^{\prime}$ since $Z\left(N, V^{\prime}, T\right)$ implicitly includes configurations for $V<V^{\prime}$. Most approaches address this deficiency by integrating over the increase in the number of states for a given volume rather the total number of states $Z(N, V, T)$. For a generalized pressure bath, this may be achieved by using a "shell" molecule whose position sets the volume of the system. However, as discussed 
in the Appendix, these overcounted states do not exist in simulations with $\mathrm{PBC}$, leaving only the simple density of volume states described by Attard. In fact, the shell particle approach is shown to be formally equivalent to Attard's density of states described below in the Appendix, not just for homogeneous systems as shown by Han and Son ${ }^{21}$ but also for $\mathrm{PBC}$ in general.

Attard builds on work by Jaynes ${ }^{22}$ describing both (i) the need for the inclusion of a density of states in the partition function when transforming a discretized sum to a continuum integral and (ii) the form of this density of states for position and scale variables based on transformation group theory. On the basis of this work, Attard ${ }^{11}$ argues that the density of volume states should be included in the NPT partition function and that this density of states is proportional to $1 / V$ as the volume sets the scale of the system. Inclusion of this density of states within the partition function integral yields

$$
Z_{N P T}=N \int_{0}^{\infty} \frac{\mathrm{d} V}{V} \mathrm{e}^{-\beta P V} \frac{V^{N}}{N ! \Lambda_{T}^{3 N}} \int \mathrm{d} \mathbf{s}^{N} \mathrm{e}^{-\beta U_{N}\left(s^{N} ; V\right)}
$$

Crucially, as Attard notes, this form of the partition function yields a range of results for the ideal gas that agree with results from the canonical ensemble, including the ideal gas law, the equivalence of chemical potential, and Gibbs free energy per particle, as well as the entropy of an ideal gas, in contrast to those derived from eq 3.

Additionally, the author noted an appealing symmetry of this partition function formulation under coordinate transformation from $V$ to the length of a cubic simulation box $L$ :

The present expression is invariant under this transformation, $d V / V \rightarrow 3 d L / L$, whereas when the density of states is neglected, the equation has a different form in the new coordinate system, $d V \rightarrow 3 L^{2} d L^{11}$

Extending this formalism to the NP $\mathrm{NT}$ ensemble also yields a partition function form invariant to a host of reasonable shape variable transforms, all unambiguously describing the shape of a tetragonal simulation box. Using this invariance, we shall easily derive a range of different MC moves, all sampling from the same partition function and therefore the same ensemble. In contrast, using the standard NPT partition function form, transformations to other cell shape control parameters lead to far less "symmetric" and intuitive forms of the partition function, as we will discuss further in section 6 .

In the following, we assume (i) a tetragonal simulation cell with $90^{\circ}$ angles between the sides of lengths $L_{\|}$and $L_{\perp}$, (ii) a bulk pressure $P$, and (iii) a lateral frame tension $\gamma$. We propose the following $\mathrm{NP} \gamma \mathrm{T}$ partition function in its most general form:

$$
\begin{aligned}
Z_{N P \gamma T} & =\int \frac{\mathrm{d} A}{A} \int \frac{\mathrm{d} V}{V} \mathrm{e}^{-\beta P V} \mathrm{e}^{\gamma A} \frac{V^{N}}{N ! \Lambda_{T}^{3 N}} \int \mathrm{d} \mathbf{s}^{N} \mathrm{e}^{-\beta U\left(\mathbf{s}^{N} ; V, A\right)} \\
& =\int \frac{\mathrm{d} A}{A} \int \frac{\mathrm{d} V}{V} \mathrm{e}^{-\beta P V} \mathrm{e}^{\gamma A} Z(N, V, A, T)
\end{aligned}
$$

using the compact notation $Z(N, V, A, T)$ for the canonical partition function in the final line. In the first line in the above equation, reduced particle coordinates $\mathbf{s}^{N}$ are employed to make the $V^{N}$ term in the partition function clear, where $\mathbf{s} \equiv\left(x / L_{\|}, y /\right.$ $\left.L_{\|}, z / L_{\perp}\right), A \equiv L_{\|}^{2}$, and $V \equiv A \cdot L_{\|}$. As both $V$ and $A$ are scale parameters of the system, each enters the partition function with a weight proportional to its inverse. We leave out the constant of proportionality, which was $N$ for the NPT partition function for a single component system, because (i) the choice of prefactor may not be immediately made by matching to an equivalent of the ideal gas law and, (ii) for the purposes of deriving Monte Carlo moves, this prefactor always cancels. One choice of prefactor may be found in the derivations in the Appendix.

2.4. Connection between Partition Function Form and Monte Carlo Algorithm. On the basis of the partition function in eq 5, we may write a pair of Monte Carlo moves designed to set $\gamma$ and $P$ by taking MC steps in $A$ and $V$. In section 3 , we present a whole range of $M C$ moves to change the simulation box shape. However, here we present $\mathrm{MC}$ moves in $A$ and $V$ in order to establish the basic approach to deriving MC moves from an NP $\mathrm{T} T$ partition function and to motivate our subsequent derivation of several equivalent forms of this partition function.

As discussed in detail in ref 23, acceptance ratios for Monte Carlo moves are constructed by requiring the flow of moves from state $o$ to state $n$ to equal the flow of moves in the opposite direction at equilibrium:

$$
p_{\text {gen }}(o \rightarrow n) \operatorname{acc}(o \rightarrow n) \mathcal{N}(o)=p_{\text {gen }}(n \rightarrow o) \operatorname{acc}(n \rightarrow o) \mathcal{N}(n)
$$

In the above, $p_{\text {gen }}$ is the probability of generating one state from the other, acc indicates the MC acceptance probability, and $\mathcal{N}$ represents the equilibrium density of states found in the integrand of the partition function. In all of the following moves, random steps in box shape are chosen from a uniform distribution, and thus $p_{\text {gen }}$ is a constant which cancels from each side of eq 6. As the integration is conducted over $\mathrm{d} A$ and $\mathrm{d} V$, Monte Carlo moves may take random steps in $A$ while holding $V$ constant, or alternately random steps in $V$ while holding $A$ constant. In conducting changes in simulation box shape, particle coordinates are rescaled such that $\mathbf{s}^{N}$ remains identical, with the consequence that for a given $A, V$, and $\mathbf{s}^{N}$

$$
\mathcal{N}\left(A, V, \mathbf{s}^{N}\right)=\frac{1}{A} \frac{1}{V} \mathrm{e}^{-\beta P V} \mathrm{e}^{\gamma A} \frac{V^{N}}{N ! \Lambda_{T}^{3 N}} \mathrm{e}^{-\beta U\left(\mathbf{s}^{N} ; V, A\right)}
$$

as indicated by the first writing of eq 5 .

Therefore, for MC moves in $V$ holding $A$ and $\mathbf{s}^{N}$ constant, we find

$$
\begin{aligned}
\frac{\operatorname{acc}\left(V_{o} \rightarrow V_{n} \mid A, \mathbf{s}^{N}\right)}{\operatorname{acc}\left(V_{n} \rightarrow V_{o} \mid A, \mathbf{s}^{N}\right)} & =\frac{\mathcal{N}\left(A, V_{n}, \mathbf{s}^{N}\right)}{\mathcal{N}\left(A, V_{o}, \mathbf{s}^{N}\right)} \\
& =\left(\frac{V_{n}}{V_{o}}\right)^{N-1} \mathrm{e}^{-\beta P \Delta V} \mathrm{e}^{-\beta \Delta U}
\end{aligned}
$$

where $\Delta V=V_{n}-V_{o}$ is the step in $V$ chosen randomly and uniformly as $\Delta V \in\left[-\left(V_{\max } / 2\right),+\left(V_{\max } / 2\right)\right] . \Delta U$ is the change in energy as $\mathbf{s}^{N}$ is held constant and therefore $\mathbf{r}^{N}$ are rescaled to accommodate the change in volume. Since $A$ is held constant, this change in volume is achieved by solely changing $L_{\perp}$ and therefore scaling the associated $z$ coordinates of each particle by $V_{n} / V_{o}$. Using the typical Metropolis expression, we therefore have

$$
\operatorname{acc}\left(V_{o} \rightarrow V_{n} \mid A, \mathbf{s}^{N}\right)=\min \left[1,\left(\frac{V_{n}}{V_{o}}\right)^{N-1} \mathrm{e}^{-\beta P \Delta V} \mathrm{e}^{-\beta \Delta U}\right]
$$

Following a nearly identical approach for Monte Carlo steps in $A$ holding $V$ and $\mathbf{s}^{N}$ constant, we find

$$
\operatorname{acc}\left(A_{o} \rightarrow A_{n} \mid V, \mathbf{s}^{N}\right)=\min \left[1, \frac{A_{o}}{A_{n}} \mathrm{e}^{\beta \gamma \Delta A} \mathrm{e}^{-\beta \Delta U}\right]
$$


In this case, as $V$ must be held constant, $L_{\|}$is rescaled by $\left(A_{n} / A_{o}\right)^{1 / 2}, L_{\perp}$ is rescaled by $A_{o} / A_{n}$, and all $\mathbf{r}^{N}$ are accordingly rescaled.

In much of the derivation above, we have been very careful in specifying the variables held constant as well as the variable over which the MC random walk is conducted. This is because these choices directly impact the form of $\mathcal{N}$ used in deriving the MC acceptance ratios. Each of these variables must be a variable of integration in the partition function from which $\mathcal{N}(o)$ and $\mathcal{N}(n)$ are drawn.

As an illustration of this, we formulate the MC acceptance ratio for a random walk sampling uniformly over $\ln V$ with $A$ and $\mathbf{s}^{N}$ held constant. Conducting a random walk in $\ln V$ has the advantage of never proposing negative trial volumes. ${ }^{24}$ However, we must rewrite eq 5 using this choice of integration variable:

$$
\begin{aligned}
Z_{N P \gamma T}= & \int \frac{\mathrm{d} A}{A} \int \mathrm{d}(\ln V) \mathrm{e}^{-\beta P V} \mathrm{e}^{\gamma A} \frac{V^{N}}{N ! \Lambda_{T}^{3 N}} \\
& \times \int \mathrm{d} \mathbf{s}^{N} \mathrm{e}^{-\beta U\left(\mathbf{s}^{N} ; V, A\right)}
\end{aligned}
$$

This immediately leads to a different $\mathcal{N}$

$$
\begin{aligned}
& \mathcal{N}\left(A, \ln V, \mathbf{s}^{N}\right) \\
& =\frac{1}{A} \mathrm{e}^{-\beta P V} \mathrm{e}^{\gamma A} \frac{V^{N}}{N ! \Lambda_{T}^{3 N}} \mathrm{e}^{-\beta U\left(\mathbf{s}^{N} ; V, A\right)}
\end{aligned}
$$

and an $\mathrm{MC}$ acceptance ratio

$$
\begin{aligned}
\operatorname{acc}\left(\ln V_{o}\right. & \left.\rightarrow \ln V_{n} \mid A, \mathbf{s}^{N}\right) \\
& =\min \left[1,\left(\frac{V_{n}}{V_{o}}\right)^{N} \mathrm{e}^{-\beta P \Delta V} \mathrm{e}^{-\beta \Delta U}\right]
\end{aligned}
$$

While this acceptance ratio appears identical to that typically used for NPT simulations, it samples our proposed $N P \gamma T$ ensemble containing the $1 / V$ density of states instead because MC steps are conducted on $\ln V$. If a simulation conducts a random walk in $V$ rather than in $\ln V$ and accepts or rejects steps based on the acceptance ratio eq 13, then the simulation does not sample the distribution of volumes $p(V)$ dictated by the two equivalent partition functions eqs 5 and 11, implying that the NPrT ensemble is not correctly sampled.

2.5. Equivalent Forms of $N P \gamma T$ Partition Function. We discussed the connection between partition function form and Monte Carlo steps and acceptance ratios because, in fact, we will construct a whole range of MC moves holding $\gamma$ and $P$ constant, yet conducting random walks in different shape parameters such as $L_{\|}$and $L_{\perp}$. Therefore, we must write various formally equivalent versions of eq 5 with pairs of $V, A, L_{\|}$, and $L_{\perp}$ as the relevant variables of integration in order to construct the correct $\mathcal{N}$ and corresponding acceptance ratios.

In order to proceed, we make the presence of $L_{\|}$and $L_{\perp}$ in the partition function eq 5 explicit rather than understood. We use delta functions such that the dimensions of the partition function are not altered:

$$
\begin{aligned}
Z_{N P \gamma T}= & \int \frac{\mathrm{d} A}{A} \int \frac{\mathrm{d} V}{V} \int \mathrm{d} L_{\|} \delta\left(L_{\|}-\sqrt{A}\right) \\
& \times \int \mathrm{d} L_{\perp} \delta\left(L_{\perp}-\frac{V}{A}\right) \mathrm{e}^{-\beta P V} \mathrm{e}^{\gamma A} Z(N, V, A, T)
\end{aligned}
$$

We now proceed to transform eq 14 into three other identical writings of $Z_{N P \gamma T}$ simply by properties of delta functions. These writings shall be crucial in developing the MC move sets in section 3.2.

We first rewrite the partition function in eq 14 such that $V$ and $L_{\|}$are the "independent" variables using the identity

$$
\delta\left(A-L_{\|}^{2}\right)=\frac{1}{2 L_{\|}}\left(\delta\left(L_{\|}-\sqrt{A}\right)+\delta\left(L_{\|}+\sqrt{A}\right)\right)
$$

Given that $L_{\|}$is constrained to be positive, we have

$$
\frac{1}{A} \delta\left(L_{\|}-\sqrt{A}\right)=\frac{2}{L_{\|}} \delta\left(A-L_{\|}^{2}\right)
$$

This then yields the partition function with $V$ and $L_{\|}$as the independent shape variables:

$$
\begin{aligned}
Z_{N P \gamma T}= & 2 \int \frac{\mathrm{d} V}{V} \int \frac{\mathrm{d} L_{\|}}{L_{\|}} \int \mathrm{d} A \delta\left(A-L_{\|}^{2}\right) \\
& \times \int \mathrm{d} L_{\perp} \delta\left(L_{\perp}-\frac{V}{L_{\|}^{2}}\right) \mathrm{e}^{-\beta P V} \mathrm{e}^{\gamma A} Z(N, V, T)
\end{aligned}
$$

On the basis of this partition function, we may derive the MC acceptance ratios $\operatorname{acc}\left(V_{o} \rightarrow V_{n} \mid L_{\|}, \mathbf{s}^{N}\right)$ and $\operatorname{acc}\left(L_{\|, o} \rightarrow L_{\|, n} \mid V, \mathbf{s}^{N}\right)$.

Alternately, using

$$
\frac{1}{A} \delta\left(L_{\perp}-\frac{V}{A}\right)=\delta\left(A L_{\perp}-V\right)=\frac{1}{L_{\perp}} \delta\left(A-\frac{V}{L_{\perp}}\right)
$$

we may rewrite eq 14 as

$$
\begin{aligned}
Z_{N P \gamma T}= & \int \frac{\mathrm{d} V}{V} \int \frac{\mathrm{d} L_{\perp}}{L_{\perp}} \int \mathrm{d} A \delta\left(A-\frac{V}{L_{\perp}}\right) \\
& \times \int \mathrm{d} L_{\|} \delta\left(L_{\|}-\sqrt{A}\right) \mathrm{e}^{-\beta P V} \mathrm{e}^{\gamma A} Z(N, V, A, T)
\end{aligned}
$$

with $V$ and $L_{\perp}$ now representing the independent shape variables. On the basis of this writing of $Z_{N P \gamma T}$, we may derive the MC acceptance ratios $\operatorname{acc}\left(V_{o} \rightarrow V_{n} \mid L_{\perp}, \mathbf{s}^{N}\right)$ and $\operatorname{acc}\left(L_{\perp, 0} \rightarrow\right.$ $\left.L_{\perp, n} \mid V, \mathbf{s}^{N}\right)$.

Finally, combining the transformation used above to yield eq 17 with the delta function identity

$$
\frac{1}{V} \delta\left(L_{\perp}-\frac{V}{L_{\|}^{2}}\right)=\frac{L_{\|}^{2}}{V} \delta\left(V-L_{\|}^{2} L_{\perp}\right)=\frac{1}{L_{\perp}} \delta\left(V-L_{\|}^{2} L_{\perp}\right)
$$

we may rewrite eq 14 as

$$
\begin{aligned}
Z_{N P \gamma T}= & 2 \int \frac{\mathrm{d} L_{\|}}{L_{\|}} \int \frac{\mathrm{d} L_{\perp}}{L_{\perp}} \int \mathrm{d} V \delta\left(V-L_{\|}^{2} L_{\perp}\right) \\
& \times \int \mathrm{d} A \delta\left(A-L_{\|}^{2}\right) \mathrm{e}^{-\beta P V} \mathrm{e}^{\gamma A} Z(N, V, T)
\end{aligned}
$$

with $L_{\|}$and $L_{\perp}$ now the independent shape variables. This formally equivalent version may be used to derive $\operatorname{acc}\left(L_{\|, 0} \rightarrow\right.$ $\left.L_{\|, n} \mid L_{\perp}, \mathbf{s}^{N}\right)$ and $\operatorname{acc}\left(L_{\perp, o} \rightarrow L_{\perp, n} \mid L_{\|}, \mathbf{s}^{N}\right)$.

We note that the completely equivalent partition functions presented in eqs 14, 17, and 19 all have a single basic form:

- Only two cell shape variables may be independently defined. We generically label these independent cell shape variables $\mathcal{L}$.

- The two "independent" cell shape variables are the two initially given shape variables of integration. For the first form in eq 14, $V$ and $A$ are these two independent variables $\mathcal{L}$. 
- In the partition function, each independent cell shape variable $\mathcal{L}$ is weighted by a density of states $1 / \mathcal{L}$. In the first case, both $\mathrm{d} V$ and $\mathrm{d} A$ enter with factors of $1 / V$ and $1 / A$.

- The remaining two cell shape variables are defined simply by integration over a single delta function. For eq 14, $L_{\|}$and $L_{\perp}$ are defined by integration over delta functions with units of inverse length, leading to the inclusion of these definitions in the partition function in a wholly normalized and unitless fashion.

Thus, regardless of the independent shape variables chosen, we may immediately write out the partition function, knowing that the Jacobian of the variable transformation has been accounted for. This is not true if the more traditional form of the NPT partition function were adhered to, as discussed later in sction 6.

\section{MONTE CARLO MOVES IN THE NPYT ENSEMBLE}

We present the Monte Carlo moves in two different subsections, the first for the two previous approaches for generating the NP $\gamma T$ outlined in section 2.2 and the second for pairs of Monte Carlo moves derived from the equivalent partition functions given in section 2.5 , explicitly stating the relevant variables of integration in each case.

In all of the shape-altering MC moves, the scaled positions of particles $\mathbf{s}^{N}$ are held constant, resulting in factors of $V^{N}$, as shown in section 2.4. We defer until section 4 a discussion of how these acceptance ratios may be altered for molecules with beads connected by bonds so that we hold the intramolecular structure constant during simulation cell reshaping moves.

3.1. Previous Approaches. 3.1.1. Old NP $\gamma T$ Moves. In our earlier work using the $N P \gamma T$ ensemble, ${ }^{9}$ we developed Monte Carlo moves based on the partition function:

$$
\begin{aligned}
Z_{N P \gamma T}= & \frac{1}{\Lambda^{3 N} N !} \frac{1}{V_{0}} \frac{1}{L_{0}} \int_{0}^{\infty} \mathrm{d} V \int_{0}^{\infty} \mathrm{d} L_{\|} V^{N} \mathrm{e}^{-\beta p V} \mathrm{e}^{\beta \gamma L_{\|}^{2}} \\
& \times \int \mathrm{d} L_{\perp} \delta\left(L_{\perp}-\frac{V}{L_{\|}^{2}}\right) \int \mathrm{d} \mathbf{s}^{N} \mathrm{e}^{-\beta U\left(s^{N} ; V, L_{\|}\right)}
\end{aligned}
$$

Moves were conducted with $V$ and $L_{\|}$as the independent variables so that when $V$ changes, $L_{\|}$and therefore $A$ do not, and no work is done against the surface tension. Likewise, when $L_{\|}$is altered, $L_{\perp}$ adjusts so that $V$ remains constant, and no work is done against pressure.

Move in $V$. We choose a step $\Delta V \in\left[-\left(V_{\max } / 2\right),\left(V_{\max } / 2\right)\right]$ yielding $V_{n}=V_{o}+\Delta V$. As $L_{\|}$and therefore $A$ are held constant, we set $L_{\perp}=V_{n} / L_{\|}^{2}$. The Monte Carlo acceptance ratio is

$$
\begin{aligned}
\operatorname{acc}\left(V_{o}\right. & \left.\rightarrow V_{n} \mid L_{\|}, \mathbf{s}^{N}\right) \\
& =\min \left[1,\left(\frac{V_{n}}{V_{o}}\right)^{N} \exp (-\beta P \Delta V-\beta \Delta U)\right]
\end{aligned}
$$

corresponding to the standard NPT Monte Carlo acceptance ratio. As in section 2.4, the conditional notation $\mid L_{\|}, \mathbf{s}^{N}$ indicates that $L_{\|}$and the scaled particle coordinates $\mathbf{s}^{N}$ are held constant.

Move in $L_{\|}$. We choose a step $\Delta L_{\|} \in\left[-\left(L_{\max } / 2\right),\left(L_{\max } / 2\right)\right]$ yielding $L_{\|, n}=L_{\|, o}+\Delta L_{\|}$. As $V$ is held constant, we set $L_{\perp, n}=V / L_{\|, n}{ }^{2}$ and $A_{n}=L_{\|, n}{ }^{2}$. The Monte Carlo acceptance ratio is

$$
\begin{aligned}
\operatorname{acc}\left(L_{\|, o}\right. & \left.\rightarrow L_{\|, n} \mid V, \mathbf{s}^{N}\right) \\
& =\min [1, \exp (\beta \gamma \Delta A-\beta \Delta U)]
\end{aligned}
$$

3.1.2. Old $N P_{\|} P_{\perp} T$ Moves. Many molecular dynamics simulations employ $P_{\perp}=P_{\|}$schemes to yield $\gamma=0$ since tension-stats for maintaining constant surface tensions are not readily available in most "packaged" molecular dynamics programs. In an attempt to replicate this approach in our Monte Carlo simulations, we apply the standard NPT Monte Carlo acceptance ratio

$$
\begin{aligned}
\operatorname{acc}\left(V_{o}\right. & \left.\rightarrow V_{n} \mid \mathbf{s}^{N}\right) \\
& =\min \left[1,\left(\frac{V_{n}}{V_{o}}\right)^{N} \exp (-\beta P \Delta V-\beta \Delta U)\right]
\end{aligned}
$$

choosing a random step in $\Delta V \in\left[-\left(V_{\max } / 2\right),\left(V_{\max } / 2\right)\right]$ and requiring that the change in $V$ results only from a scaling of $L_{\perp}$ or only from scaling of $L_{\|}$

3.2. New Equivalent Monte Carlo Moves. Crucially, the entirely equivalent writings of $Z_{N P \gamma T}$ given in section 2.5 allow us to conceive of a whole host of different Monte Carlo move pairs to represent a set $P$ and a set $\gamma$. A pair of MC moves must allow variation of shape both laterally and normally in order to probe all relevant shapes for a set $\gamma$ and $P$. We present below four pairs of moves that do this, but others may be conceived of as well.

In constructing the acceptance ratios given below, we are quite explicit in which "independent" shape variable is varied in each case as well as which "independent" shape variable is held constant. This specificity is required, as these choices impact the Monte Carlo acceptance ratios, as discussed for changing between a random walk in $V$ and a random walk in $\ln V$ in section 2.4.

3.2.1. Steps in $V$ and $L_{\|}$. The first Monte Carlo move set is based on the partition function writing in eq 17 and is the closest analog to the moves described in section 3.1.1 for $N P_{\perp} \gamma T$ simulations. ${ }^{9}$ We may either step uniformly and randomly in $V$ with $L_{\|}$constant or step uniformly and randomly in $L_{\|}$with $V$ constant.

Move in $\boldsymbol{V}$ holding $L_{\|}$constant. We choose a step $\Delta V \in$ $\left[-\left(V_{\max } / 2\right),\left(V_{\max } / 2\right)\right]$ yielding $V_{n}=V_{o}+\Delta V$. As $L_{\|}$and therefore $A$ are held constant, we set $L_{\perp}=V_{n} / L_{\|}^{2}$. The Monte Carlo acceptance ratio is

$$
\begin{aligned}
\operatorname{acc}\left(V_{o}\right. & \left.\rightarrow V_{n} \mid L_{\|}, \mathbf{s}^{N}\right) \\
& =\min \left[1,\left(\frac{V_{n}}{V_{o}}\right)^{N-1} \exp (-\beta P \Delta V-\beta \Delta U)\right]
\end{aligned}
$$

Move in $L_{\|}$holding $V$ constant. We choose a step $\Delta L_{\|} \in$ $\left[-\left(L_{\max } / 2\right),\left(L_{\max } / 2\right)\right]$ yielding $L_{\|, n}=L_{\|, 0}+\Delta L_{\|}$. As $V$ is held constant, we set $L_{\perp}=V_{n} / L_{\|, n}{ }^{2}$ and $A_{n}=L_{\|, n}{ }^{2}$. The Monte Carlo acceptance ratio is

$$
\operatorname{acc}\left(L_{\|, o} \rightarrow L_{\|, n} \mid V, \mathbf{s}^{N}\right)=\min \left[1, \frac{L_{\|, o}}{L_{\|, n}} \exp (\beta \gamma \Delta A-\beta \Delta U)\right]
$$

The two acceptance ratios given above differ from those previously employed by a factor of $V_{o} / V_{n}$ and $L_{\|, o} / L_{\|, n}$. 
3.2.2. Steps in $L_{\|}$and $L_{\perp}$. The second Monte Carlo move set is based on the partition function form in eq 21 and is the closest analog to molecular dynamics implementations using a pressure tensor and decoupled moves in $L_{\perp}$ and $L_{\|}$. We may either step uniformly and randomly in $L_{\perp}$ with $L_{\|}$constant or step uniformly and randomly in $L_{\|}$with $L_{\perp}$ constant.

Move in $L_{\|}$Holding $L_{\perp}$ Constant. We choose a step $\Delta L_{\|} \in$ $\left[-\left(L_{\|, \max } / 2\right),\left(L_{\|, \max } / 2\right)\right]$ yielding $L_{\|, n}=L_{\|, o}+\Delta L_{\|}$. As $L_{\perp}$ is held constant, we set $V_{n}=L_{\perp} \cdot L_{\|, n}{ }^{2}$ and $A_{n}=L_{\|, n}{ }^{2}$. The Monte Carlo acceptance ratio is

$$
\begin{aligned}
\operatorname{acc}\left(L_{\|, o} \rightarrow\right. & \left.L_{\|, n} \mid L_{\perp}, \mathbf{s}^{N}\right)=\min \left[1,\left(\frac{A_{n}}{A_{o}}\right)^{N-1 / 2}\right. \\
& \times \exp (-\beta P \Delta V+\beta \gamma \Delta A-\beta \Delta U)]
\end{aligned}
$$

Move in $L_{\perp}$ Holding $L_{\|}$Constant. We choose a step $\Delta L_{\perp} \in$ $\left[-\left(L_{\perp, \max } / 2\right),\left(L_{\perp, \max } / 2\right)\right]$ yielding $L_{\perp, n}=L_{\perp, o}+\Delta L_{\perp}$. As $L_{\|}$and therefore $A$ are held constant, we set $V_{n}=L_{\perp, n} \cdot L_{\|, n}{ }^{2}$. The Monte Carlo acceptance ratio is

$$
\begin{aligned}
\operatorname{acc}\left(L_{\perp, o}\right. & \left.\rightarrow L_{\perp, n} \mid L_{\|}, \mathbf{s}^{N}\right) \\
& =\min \left[1,\left(\frac{L_{\perp, n}}{L_{\perp, o}}\right)^{N-1} \exp (-\beta P \Delta V-\beta \Delta U)\right]
\end{aligned}
$$

3.2.3. Steps in $V$ with $L_{\|}$Constant and in $V$ with $L_{\perp}$ Constant $\left(V \mid L_{\|}\right.$and $\left.V \mid L_{\perp}\right)$. This third Monte Carlo move set involves alternating between two different expressions for $Z_{N P \gamma T}$, taking steps in $V$ and holding a different box side constant. Building on the partition functions in eq 17 and in eq 19, we have two ways to step uniformly in $V$.

Move in $V$ Holding $L_{\|}$Constant. This move has an acceptance ratio identical to that described in eq 26 :

$$
\begin{aligned}
\operatorname{acc}\left(V_{o}\right. & \left.\rightarrow V_{n} \mid L_{\|}, \mathbf{s}^{N}\right) \\
& =\min \left[1,\left(\frac{V_{n}}{V_{o}}\right)^{N-1} \exp (-\beta P \Delta V-\beta \Delta U)\right]
\end{aligned}
$$

Move in $\boldsymbol{V}$ Holding $L_{\perp}$ Constant. We choose a step $\Delta V \in$ $\left[-\left(V_{\max } / 2\right),\left(V_{\max } / 2\right)\right]$ yielding $V_{n}=V_{o}+\Delta V$. As $L_{\perp}$ is held constant, we set $L_{\|, n}=\left(V / L_{\perp}\right)^{1 / 2}$ and $A_{n}=L_{\|, n}{ }^{2}$. The Monte Carlo acceptance ratio is

$$
\begin{aligned}
\operatorname{acc}\left(V_{o} \rightarrow\right. & \left.V_{n} \mid L_{\perp}, \mathbf{s}^{N}\right)=\min \left[1,\left(\frac{V_{n}}{V_{o}}\right)^{N-1}\right. \\
& \times \exp (-\beta P \Delta V+\beta \gamma \Delta A-\beta \Delta U)]
\end{aligned}
$$

This combination of MC steps is the analog of the move set described in section 3.1.2 as an implementation of $P_{\perp}=P_{\|}$for yielding $\gamma=0$. A distinct advantage here is that nonzero surface tension is also easily simulated.

3.2.4. Steps in $V$ and $L_{\perp}$. This combination of MC moves is perhaps the most counterintuitive, as we typically would consider both $L_{\perp}$ and $V$ to be conjugate to $P$. However, we may quite validly write $\mathrm{MC}$ moves choosing these two "independent" shape parameters as the cell shape is allowed to vary both laterally and normally through the application of both moves.

Move in $\boldsymbol{V}$ Holding $L_{\perp}$ Constant. This move has an acceptance ratio identical to that given in eq 30 :

$$
\begin{aligned}
\operatorname{acc}\left(V_{o} \rightarrow\right. & \left.V_{n} \mid L_{\perp}, \mathbf{s}^{N}\right)=\min \left[1,\left(\frac{V_{n}}{V_{o}}\right)^{N-1}\right. \\
& \times \exp (-\beta P \Delta V+\beta \gamma \Delta A-\beta \Delta U)]
\end{aligned}
$$

Move in $L_{\perp}$ Holding $V$ Constant. We choose a step $\Delta L_{\perp} \in$ $\left[-\left(L_{\max } / 2\right),\left(L_{\max } / 2\right)\right]$ yielding $L_{\perp, n}=L_{\perp, o}+\Delta L_{\perp}$. As $V$ is held constant, we set $L_{\|, n}=\left(V / L_{\perp}\right)^{1 / 2}$ and $A_{n}=V / L_{\perp}$. The Monte Carlo acceptance ratio is

$$
\begin{aligned}
\operatorname{acc}\left(L_{\perp, o}\right. & \left.\rightarrow L_{\perp, n} \mid V, \mathbf{s}^{N}\right) \\
& =\min \left[1, \frac{L_{\perp, o}}{L_{\perp, n}} \exp (\beta \gamma \Delta A-\beta \Delta U)\right]
\end{aligned}
$$

3.2.5. Simplified Acceptance Ratios with Steps over $\ln \mathcal{L}$. Finally, we note that all Monte Carlo acceptance ratios outlined above become much simpler if the random walk over each variable is conducted using the natural logarithm of the shape variable. In each case, the acceptance ratio would be

$$
\begin{aligned}
\operatorname{acc}(o \rightarrow n)=\min & {\left[1,\left(\frac{V_{n}}{V_{o}}\right)^{N}\right.} \\
& \times \exp (-\beta P \Delta V+\beta \gamma \Delta A-\beta \Delta U)]
\end{aligned}
$$

The collapse of all acceptance ratios onto this form when employing the natural logarithm appears to be a consequence of the rectilinear nature of the simulation box. Therefore, for general simulation box shapes, this single form likely is not valid. Furthermore, we do not simulate with this acceptance ratio combined with steps in the logarithm of the shape variables because the simplicity of the final acceptance ratio form might easily obscure the care required in correctly constructing shape-changing Monte Carlo moves a priori. However, the simplicity of this form as well as the fact that negative shape variables are automatically disallowed by moves in the logarithm suggest that conducting shape moves in general using the natural logarithm could be quite advantageous.

\section{LIPID MODEL AND SIMULATION DETAILS}

In order to test the Monte Carlo move sets detailed in the previous section, we simulate a coarse-grained lipid bilayer system.

Our lipid model employs soft repulsive interactions between three types of beads-water (w), lipid hydrophilic head (h), and lipid hydrophobic tail $(\mathrm{t})$ - as described in great detail in previous publications from our group. ${ }^{25,26}$ We simulate the lipid DMPC with two symmetric saturated tails, represented by $h_{3}\left(t_{4}\right)_{2}$.

We employ a hybrid dissipative particle dynamics (DPD)Monte Carlo scheme with each MC cycle consisting of either shape-changing MC moves or short DPD trajectories, as described in previous publications. ${ }^{9}$ The dissipative particle 
dynamics (DPD) trajectories propagate the coordinates of the lipids and water beads forward in time with a well-defined thermostat that obeys the Boltzmann distribution. ${ }^{27}$ These trajectories vary in length uniformly from 1 time step to 50 time steps with $\Delta t=0.03$. Within the overall hybrid DPD-MC scheme, DPD trajectories are generated with $20 \%$ likelihood, and each of the two MC moves within a given pair of shape changing moves are attempted $40 \%$ of the time.

As discussed in ref 9, when conducting shape changes, we only scale the position $\mathbf{r}$ of one bead in each molecule and hold all relative intramolecular positions of the remaining $n$ sites constant, $\mathcal{R}^{n}$. This, therefore, suggests a writing of the NP $\mathrm{N} T$ partition function as

$$
\begin{aligned}
Z_{N P \gamma T}= & \int \frac{\mathrm{d} A}{A} \int \frac{\mathrm{d} V}{V} \mathrm{e}^{-\beta P V} \mathrm{e}^{\gamma A} \frac{V^{N_{\text {mol }}} q(T)^{N_{\text {lip }}}}{N ! \Lambda_{T}^{3 N_{\mathrm{w}}}} \\
& \times \int \mathrm{d} \mathbf{s}^{N_{\text {mol }}} \int \mathrm{d} \mathcal{R}^{n \cdot N_{\text {lip }}} \mathrm{e}^{-\beta U\left(s^{N_{\text {mol }}}, \mathcal{R}^{n \cdot N_{\text {lip }} V, A}\right)}
\end{aligned}
$$

where $N_{\text {mol }} \equiv N_{\text {lip }}+N_{\text {w }}$ and the kinetic contribution to the partition function for each lipid molecule has been expressed as $q(T)$ while that for the single-site water beads has remained $\Lambda_{T}{ }^{-3}$. Consequently, each $N$ in the Monte Carlo acceptance ratios given in section 3 actually corresponds to $N_{\text {mol }}$ as only a total of $N_{\text {mol }}$ positions are rescaled during any of the MC moves and the remaining $n \cdot N_{\text {lip }}$ relative intramolecular positions are held constant. For the lipid model considered herein, where the spring constants associated with the bonds are moderate, holding the intramolecular relative positions constant presents no dramatic advantage over scaling the positions of all beads within the molecule. However, for all-atom models or to a lesser extent for the DPD cholesterol and protein models, the overall bond stiffness is greater and the scaling of the coordinates of all beads leads to a substantial increase in intermolecular bonding energies and consequently samples the important phase space less efficiently than holding the intramolecular coordinates constant.

We run a range of simulations with $N_{\text {lip }}$ either set to 16 or 256 and $N_{\mathrm{w}}$ adjusted in order to maintain full bilayer hydration with a ratio of $N_{\mathrm{w}} / N_{\text {lip }}=25$. Ten separate simulations with $N_{\text {lip }}=256$ are run for $2 \times 10^{5}$ DPD-MC cycles. Excluding the first $25 \%$ of each simulation for equilibration, a total of $1.5 \times$ $10^{6} \mathrm{MC}$ cycles are used for data collection. Ten simulations with $N_{\text {lip }}=16$ are equilibrated for $2 \times 10^{5}$ cycles each and then run for a further $2 \times 10^{7} \mathrm{DPD}-\mathrm{MC}$ cycles, resulting in $2 \times 10^{8}$ $\mathrm{MC}$ cycles used to yield highly converged histograms. Both choices for bilayer size are quite small, as these small systems make the collection of large data sets feasible. Additionally, differences arising from inequivalent ensembles are more easily detected in smaller system sizes. We expect that the average shape quantities may differ between $N_{\text {lip }}=256$ and $N_{\text {lip }}=16$, as the latter system is quite small, and both pinning of surface fluctuation modes at the boundaries as well as other finite size effects will impact the averages. However, for a single $N_{\text {lip }}$, these considerations should not affect the agreement between MC move sets, provided that the same ensemble is sampled.

Surface tensions of both $\gamma=0.0$ and $\gamma=2.0$ are examined, while pressure is maintained at $P=22.28$, the rescaled ambient pressure for our DPD model as explained in ref 9 .

In order to gauge the agreement between different $\mathrm{MC}$ move sets, we examine the distributions of three simulation box shape parameters: (i) the volume per lipid molecule, $V_{\text {lip }} \equiv V / N_{\text {lip }}$; (ii) the bilayer area per lipid molecule $A_{\text {lip }} \equiv A /\left(N_{\text {lip }} / 2\right)$; and (iii) $L_{\perp}$. We do not remove the water volume from $V_{\text {lip }}$, so this quantity is actually the volume of one lipid and 25 water beads. We also consider the values of $P_{\perp}, P_{\|}$, and $\gamma$ resulting from the simulations to demonstrate that the set quantities are reproduced.

All quantities are reported in the reduced units of the DPD model.

\section{RESULTS FOR EQUIVALENCE BETWEEN MC SCHEMES}

A reasonable initial check on the equivalence of these simulation approaches is examining the average values and average fluctuations of cell shape parameters as well as surface tension and pressure for 256 lipids at both surface tension values $(\gamma=$ 0.0 and $\gamma=2.0)$. We report results from each of the six sets of $\mathrm{MC}$ moves that alter cell shape described in section 3. All shape results are given in Tables 1 and 3, and results for pressure and

Table 2. Average Thermodynamic Fields for $\boldsymbol{P}=\mathbf{2 2 . 2 8}$ and $\gamma=0.0$ and $N_{\text {lip }}=256$

$\begin{array}{lccc}\text { MC move set } & \left\langle P_{\perp}\right\rangle^{a} & \left\langle P_{\|}\right\rangle^{a} & \langle\gamma\rangle^{a} \\ V \text { and } L_{\|} & 22.270\left(1.3 \times 10^{-3}\right) & 22.269\left(1.1 \times 10^{-3}\right) & 0.018(0.022) \\ L_{\|} \text {and } L_{\perp} & 22.268\left(1.1 \times 10^{-3}\right) & 22.268\left(0.7 \times 10^{-3}\right) & 0.006(0.020) \\ V \mid L_{\|} \text {and } V \mid L_{\perp} & 22.270\left(1.5 \times 10^{-3}\right) & 22.268\left(0.9 \times 10^{-3}\right) & 0.028(0.027) \\ V \text { and } L_{\perp} & 22.271\left(2.1 \times 10^{-3}\right) & 22.268\left(1.2 \times 10^{-3}\right) & 0.036(0.016) \\ \text { old } N P \gamma T & 22.272\left(1.3 \times 10^{-3}\right) & 22.269\left(1.0 \times 10^{-3}\right) & 0.047(0.013) \\ \text { old } N P_{\|} P_{\perp} T & 22.270\left(1.5 \times 10^{-3}\right) & 22.269\left(0.9 \times 10^{-3}\right) & 0.010(0.022) \\ { }^{a} \text { Calculated errors in parentheses. } & & \end{array}$

surface tensions are in Tables 2 and 4 . The old $N P_{\perp} P_{\|} T$ scheme is only employed for $\gamma=0.0$ since typical implementations of this approach do not specify a route for variation of $\gamma$.

All cell shape averages agree up to the fourth significant figure, and their fluctuations agree up to the second significant figure; quantities associated with $V_{\text {lip }}$ are converged up to one additional significant figure. Surface tensions agree to the second decimal place, and the pressures agree to the fifth significant figure. Overall, this agreement between both the old MC schemes as well as the complement of new MC schemes is quite good.

This agreement alone is an important result for practical applications. Typical simulation systems are larger than $N_{\text {lip }}=256$, particularly for coarse-grained models, and so any differences arising due to differences between an exponent of $N$ and of $N-1$ are negligibly small for the accuracy sought in such

Table 1. Average Shape and Fluctuations for $P=22.28$, and $\gamma=0.0$, and $N_{\text {lip }}=256$

$\begin{array}{lcccccc}\text { MC move set } & \left\langle V_{\text {lip }}\right\rangle & \left\langle A_{\text {lip }}\right\rangle & \left\langle L_{\perp}\right\rangle & \left(\left\langle\delta V_{\text {lip }}{ }^{2}\right\rangle\right)^{1 / 2} & \left(\left\langle\delta A_{\text {lip }}{ }^{2}\right\rangle\right)^{1 / 2} & \left(\left\langle\delta L_{\perp}{ }^{2}\right\rangle\right)^{1 / 2} \\ V \text { and } L_{\|} & 11.9012 & 1.5567 & 15.293 & 0.0264 & 0.0206 & 0.199 \\ L_{\|} \text {and } L_{\perp} & 11.9010 & 1.5567 & 15.292 & 0.0266 & 0.0199 & 0.193 \\ V \mid L_{\|} \text {and } V \mid L_{\perp} & 11.9010 & 1.5574 & 15.285 & 0.0265 & 0.0200 & 0.193 \\ V \text { and } L_{\perp} & 11.9012 & 1.5575 & 15.285 & 0.0265 & 0.0202 & 0.195 \\ \text { old } N P \gamma T & 11.9013 & 1.5575 & 15.285 & 0.0264 & 0.0201 & 0.194 \\ \text { old } N P_{\|} P_{\perp} T & 11.9011 & 1.5579 & 15.281 & 0.0265 & 0.0202 & 0.194\end{array}$


Table 3. Average Shape and Fluctuations for $P=22.28$ and $\gamma=2.0$ and $N_{\text {lip }}=256$

\begin{tabular}{lccccc} 
MC move set & $\left\langle V_{\text {lip }}\right\rangle$ & $\left\langle A_{\text {lip }}\right\rangle$ & $\left\langle L_{\perp}\right\rangle$ & $\left(\left\langle\delta V_{\text {lip }}^{2}\right\rangle\right)^{1 / 2}$ & $\left(\left\langle\delta A_{\text {lip }}^{2}\right\rangle\right)^{1 / 2}$ \\
$V$ and $L_{\|}$ & 11.9440 & 1.7477 & 13.671 & 0.0265 & 0.0251 \\
$L_{\|}$and $L_{\perp}$ & 11.9444 & 1.7479 & 13.670 & 0.0266 & 0.0255 \\
$V \mid L_{\|}$and $V \mid L_{\perp}$ & 11.9439 & 1.7460 & 13.684 & 0.0266 & 0.191 \\
$V$ and $L_{\perp}$ & 11.9444 & 1.7497 & 13.656 & 0.0266 & 0.0246 \\
old $N P \gamma T$ & 11.9439 & 1.7463 & 13.682 & 0.0267 & 0.189 \\
\hline
\end{tabular}

Table 4. Average Thermodynamic Fields for $P=22.28$ and $\gamma=2.0$ and $N_{\text {lip }}=256$

\begin{tabular}{lccc} 
MC move set & $\left\langle P_{\perp}\right\rangle^{a}$ & $\left\langle P_{\|}\right\rangle^{a}$ & $\langle\gamma\rangle^{a}$ \\
$V$ and $L_{\|}$ & $22.272\left(1.3 \times 10^{-3}\right)$ & $22.123\left(1.7 \times 10^{-3}\right)$ & $2.045(0.017)$ \\
$L_{\|}$and $L_{\perp}$ & $22.271\left(1.4 \times 10^{-3}\right)$ & $22.121\left(0.6 \times 10^{-3}\right)$ & $2.036(0.014)$ \\
$V \mid L_{\|}$and $V \mid L_{\perp}$ & $22.270\left(1.2 \times 10^{-3}\right)$ & $22.123\left(0.6 \times 10^{-3}\right)$ & $2.002(0.016)$ \\
$V$ and $L_{\perp}$ & $22.273\left(1.3 \times 10^{-3}\right)$ & $22.123\left(1.0 \times 10^{-3}\right)$ & $2.040(0.016)$ \\
old $N P \gamma T$ & $22.270\left(1.4 \times 10^{-3}\right)$ & $22.124\left(1.4 \times 10^{-3}\right)$ & $1.997(0.015)$ \\
${ }^{a}$ Calculated errors in parentheses. & & \\
\hline
\end{tabular}

simulations. Furthermore, schemes decoupling the lateral and normal cell volume changes at set $P$ yield results similar to all other $\gamma=0$ schemes. Finally, using the formalism outlined in sections $2.3-2.5$ and 3.2, such approaches are easily modified to allow for $\gamma \neq 0$, as demonstrated by the numerical agreement of the new MC move sets at $\gamma=2.0$.

However, we also seek a more detailed understanding of the equivalence of various MC moves. Histograms of sampled $V_{\text {lip, }} A_{\text {lip }}$, and $L_{\perp}$ provide a more complete view of the shape fluctuations sampled by each MC scheme. Such fluctuations will only be identical when the MC schemes exactly sample the same ensemble.

The histograms for each of the six simulated MC move sets, the two old and the four new, are very similar for both surface tensions with $N_{\text {lip }}=256$. Any differences between the histograms are well within the error bars of the collected histograms, as shown in the Supporting Information (SI) in Figures S1-S6. This similarity is expected since the four new move sets are formally equivalent, and differences with the old move sets should be negligible for large system sizes, as has been argued by other authors. ${ }^{11,28}$ The volume histograms are better converged than those for the area and box length fluctuations, but agreement is reasonable for all.

In order to reveal the equivalence or inequivalence between various $\mathrm{MC}$ move sets, we simulate a very small lipid bilayer system with $N_{\text {lip }}=16$ and $N_{\mathrm{w}}=400$ at $\gamma=0.0$ and $\gamma=2.0$ for a total of $2 \times 10^{8}$ cycles. The small system is crucial for determining which histogram differences are statistically significant. Not only are such differences only substantial at small system sizes, but also the small system size makes such extensive data collection computationally reasonable. The probability density distributions for $V_{\text {lip }}, A_{\text {lip }}$, and $L_{\perp}$ at $\gamma=0.0$ are given in Figures 2-4. The lower panels in each plot display the deviations of each probability density profile from the "best" estimate. The four new MC moves sets outlined in section 3.2 were formally derived to sample from the same ensemble, so we construct the best estimate from a weighted average of their probability density profiles, with each histogram point weighted by the inverse variance at that point.

For volume, the histograms for the four new MC move sets given in section 3.2 are identical to one another and the two old MC move sets given in section 3.1 are also identical to each other. Within each of these classes the sampled NPT partition function is identical, and between the two classes, the sampled

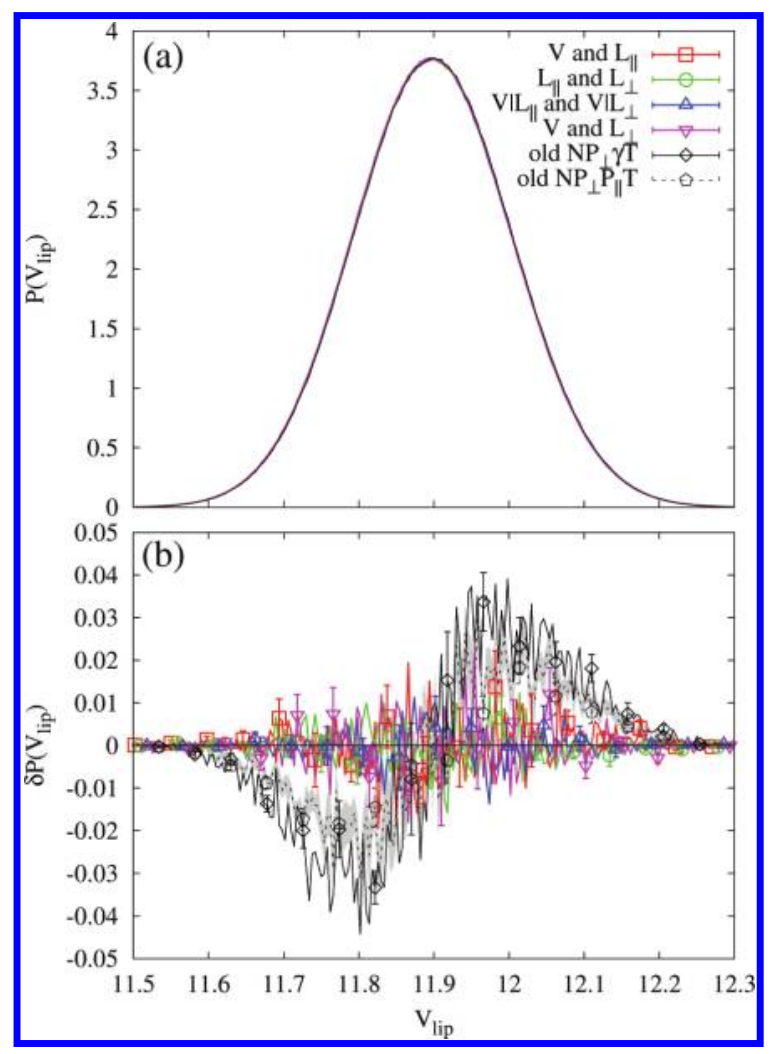

Figure 2. Volume probability density and deviations in a and $b$, respectively, for $N_{\text {lip }}=16$ and $\gamma=0.0$. Displayed data symbols are staggered across MC move sets and only shown every 12 points for readability.

NPT partition function differs by a factor of $V$. As such, these results are entirely expected.

For $L_{\perp}$ and $A$, the equivalence between the four new MC move sets is maintained as expected. However, the two old MC move sets are no longer equivalent to one another in their shape fluctuations. The old $N P \gamma T$ and $N P_{\|} P_{\perp} T$ Monte Carlo schemes are distinctly inequivalent. This also indicates that these two schemes have different correlations between $L_{\perp}$ and $A$, thus allowing for different $p\left(L_{\perp}\right)$ and $p(A)$ while having identical $p(V)$. Interestingly, the old $N P_{\|} P_{\perp} T$ Monte Carlo scheme generates shape fluctuations numerically identical to those of the new schemes, though we do not believe any deep significance should be ascribed to this similarity.

Results for $\gamma=2.0$ are shown in Figures 5-7. The observations for equivalence and inequivalence between various schemes for $\gamma=0.0$ hold equally well for nonzero surface tension. The sole distinction lies in the fact there is no definition of the old $N P_{\|} P_{\perp} T$ Monte Carlo scheme for nonzero surface tension. But the closest analog among our newly defined MC schemes, moves in $V$ holding $L_{\perp}$ constant and moves in $V$ holding $L_{\|}$constant, 

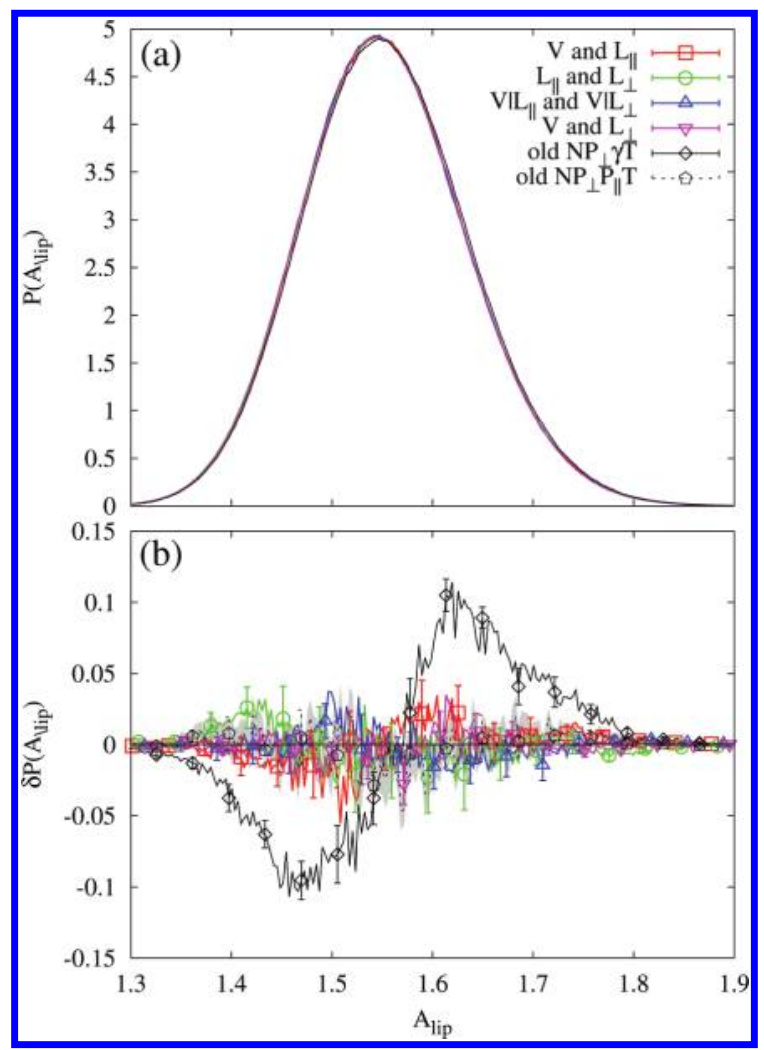

Figure 3. Area probability density and deviations in a and $b$, respectively, for $N_{\text {lip }}=16$ and $\gamma=0.0$. Displayed data symbols are staggered across MC move sets and only shown every 12 points for readability.

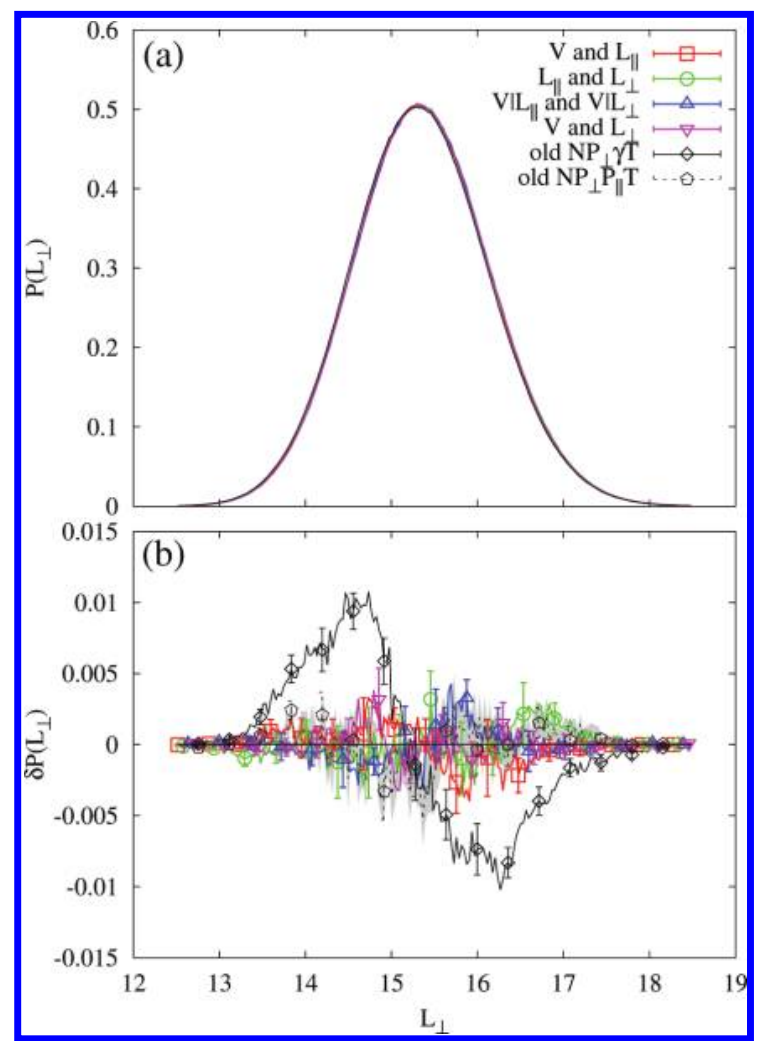

Figure 4. $L_{\perp}$ probability density and deviations in a and $\mathrm{b}$, respectively, for $N_{\text {lip }}=16$ and $\gamma=0.0$. Displayed data symbols are staggered across MC move sets and only shown every 12 points for readability.

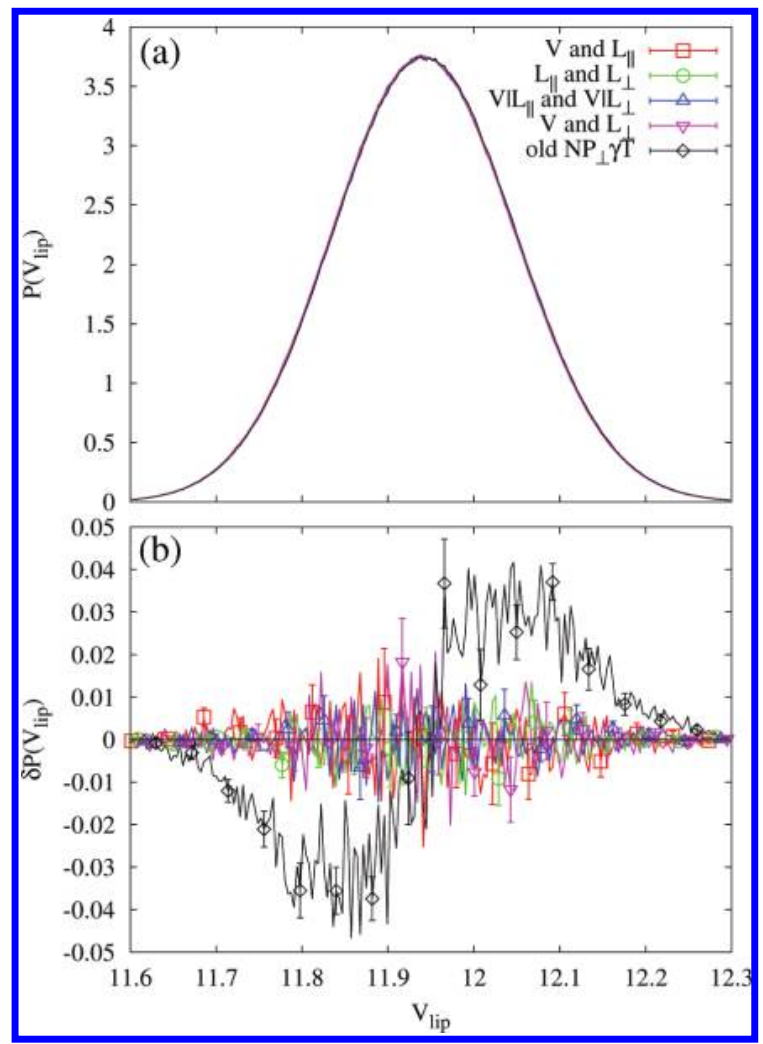

Figure 5. Volume probability density and deviations in a and $b$, respectively, for $N_{\text {lip }}=16$ and $\gamma=2.0$. Displayed data symbols are staggered across MC move sets and only shown every 12 points for readability.

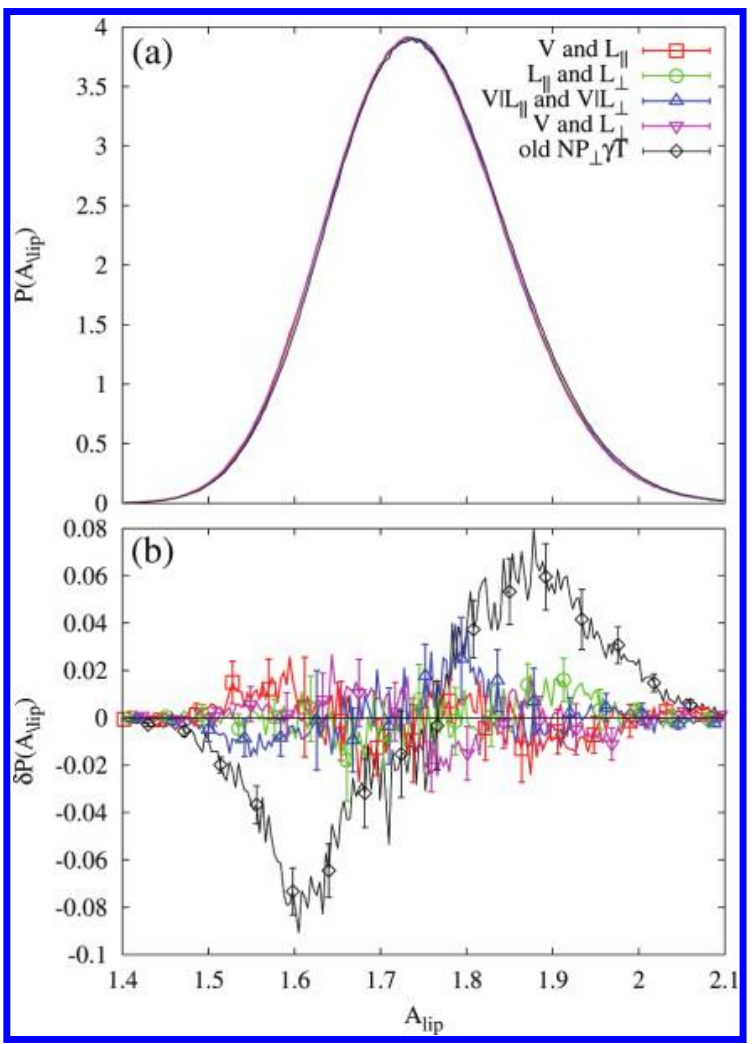

Figure 6. Area probability density and deviations in a and b, respectively, for $N_{\text {lip }}=16$ and $\gamma=2.0$. Displayed data symbols are staggered across MC move sets and only shown every 12 points for readability. 


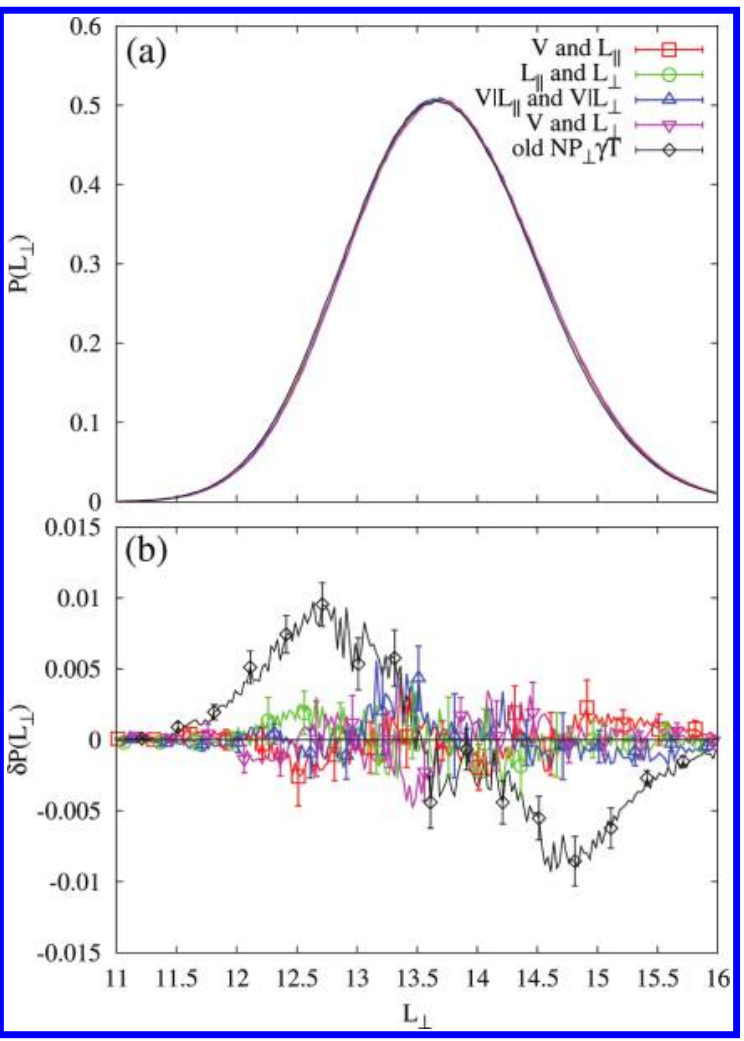

Figure 7. $L_{\perp}$ probability density and deviations in a and $\mathrm{b}$, respectively, for $N_{\text {lip }}=16$ and $\gamma=2.0$. Displayed data symbols are staggered across MC move sets and only shown every 12 points for readability.

does display numerical equivalence with all the other new MC schemes, as the derivation would suggest.

\section{DISCUSSION OF TRADITIONAL NP $\gamma T$ FORMULATION}

We may better understand why the two old schemes for $\gamma=0.0$ are only identical in their volume fluctuations and not their shape fluctuations by attempting the independent variable transformations as done in section 2.5 for a general $N P \gamma T$ partition function with the volume and area length scale factors as constant prefactors. We begin with

$$
\begin{aligned}
Z_{N P \gamma T}= & \frac{1}{A_{0} V_{0}} \int \mathrm{d} A \int \mathrm{d} V \int \mathrm{d} L_{\|} \delta\left(L_{\|}-\sqrt{A}\right) \\
& \times \int \mathrm{d} L_{\perp} \delta\left(L_{\perp}-\frac{V}{A}\right) \mathrm{e}^{-\beta P V} \mathrm{e}^{\gamma A} Z(N, V, A, T)
\end{aligned}
$$

The equivalent of our old NP $\mathrm{T}$ move attempts are generated with $V$ and $L_{\|}$as the independent variables. This transformation of independent variables by the delta function identity eq 16 yields

$$
\begin{aligned}
Z_{N P \gamma T}= & \frac{2}{A_{0} V_{0}} \int \mathrm{d} L_{\|} L_{\|} \int \mathrm{d} V \int \mathrm{d} A \delta\left(A-L_{\|}^{2}\right) \\
& \times \int \mathrm{d} L_{\perp} \delta\left(L_{\perp}-\frac{V}{A}\right) \mathrm{e}^{-\beta P V} \mathrm{e}^{\gamma A} Z(N, V, A, T)
\end{aligned}
$$

Therefore, the MC moves in volume are still sampled by the old MC acceptance ratio in eq 23. However, the acceptance ratio for $\mathrm{MC}$ moves in $L_{\|}$given in eq 24 should be altered by an additional factor of $L_{\|, n} / L_{\|, o}$.

This suggests an error in the NP $\mathrm{T} T$ ensemble we have used in previous publications. ${ }^{9}$ Rather, we may show that the partition function previously employed by Smit and co-workers ${ }^{3}$ for the
NV $\gamma \mathrm{T}$ ensemble:

$$
Z_{N V \gamma T}=\int \mathrm{d} \lambda \mathrm{e}^{\gamma A} Z(N, V, A, T)
$$

may be made consistent with either the old $N P \gamma T$ formulation in section 3.1 or with our new NP $\mathrm{T}$ T formalism in section 2.5 instead of with the NP $\gamma T$ partition function outlined above in eq 34. If, as in ref 8 , we define $\lambda \equiv L_{\|} / L_{0}$, then $d \lambda=d L_{\|} / L_{0}$, making eq 36 extensible to the $N P \gamma T$ formulation in eq 22 . If instead we map $L_{\|, o} \rightarrow L_{\|, n}=\lambda \cdot L_{\|, o}$ as in ref 3 , then $d \lambda=d L_{\|} / L_{\|}$, resulting in the partition function

$$
\begin{aligned}
Z_{N V \gamma T}= & \int \frac{\mathrm{d} L_{\|}}{L_{\|}} \int \mathrm{d} L_{\perp} \delta\left(L_{\perp}-\frac{V}{L_{\|}^{2}}\right) \\
& \times \int \mathrm{d} A \delta\left(A-L_{\|}^{2}\right) \mathrm{e}^{\gamma A} Z(N, V, A, T)
\end{aligned}
$$

with all shape variable relationships made explicit. This $N V \gamma T$ partition function is easily extended to yield the NP $\mathrm{T}$ ensemble given in eq 17 . Thus, several previously proposed schemes for MC moves in shape transformations in fact sample slightly different ensembles.

Furthermore, transformation of eq 34 to sample the $N P_{\|} P_{\perp} T$ move set also yields an unexpected partition function form counter to the intuition of simple volume moves in the lateral and normal directions. Moves in volume only in the normal direction and therefore holding the lateral shape constant are best derived again from eq 35, yielding the same acceptance ratio eq 23. However, moves in volume holding the normal length constant are best derived by rewriting eq 34 so that $V$ and $L_{\perp}$ are the independent variables. Using the delta function identity in eq 18 , we find

$$
\begin{aligned}
Z_{N P \gamma T}= & \frac{1}{A_{0} V_{0}} \int \mathrm{d} L_{\perp} \int \mathrm{d} V \frac{V}{L_{\perp}^{2}} \int \mathrm{d} A \delta\left(A-\frac{V}{L_{\perp}}\right) \\
& \times \int d L_{\|} \delta\left(L_{\|}-\sqrt{A}\right) \mathrm{e}^{-\beta P V} \mathrm{e}^{\gamma A} Z(N, V, A, T)
\end{aligned}
$$

This yields the surprising modification of the acceptance ratio in eq 25 by an additional, unexpected factor of $V_{n} / V_{o}$.

As the original acceptance ratios given in section 3.1 yielded quite reasonable results for simulation box volume and shape for $N_{\text {lip }}=256$, we observe that these prefactors have little effect on observed quantities for larger systems and, as such, are still reasonable approaches.

These derivations do highlight that using a form of the partition function as in eq 34 with volume and area scales outside of the integral yields a partition function with highly asymmetric and nontrivial Jacobian transformations. This then requires great care in the construction of partition functions with different independent variables as indicated by Martyna and co-workers when deriving fully flexible simulation cell protocols for constant pressure simulations. ${ }^{29,30}$ Building on the previously proposed $Z_{N V \gamma T}$ employed by our research group also requires care, as different reasonable definitions of the shape transformation variable $\lambda$ result in two different partition functions.

Instead, using a partition function based on the form suggested by Attard ${ }^{11}$ with the volume and area scales internal to the integral yields a partition function with remarkable symmetry to all shape variable transformations as demonstrated in our derivations in section 2.5. Furthermore, this partition function form accounts for the correct transformation of the volume and area sums to continuous integration ${ }^{11}$ as well as 
correctly weighting volume and area states as proven in the Appendix.

\section{CONCLUSIONS}

In this paper, we have derived and implemented a range of Monte Carlo moves designed to sample a constant surface tension and constant pressure. With care in transforming among the variables which can describe the shape of the simulation box$V, A, L_{\|}$, and $L_{\perp}$-we derive a set of MC moves that all sample the identical ensemble and yet tune different pairs of shape variables.

Formally, we find that the analog of the NPT partition function given by Attard is most advantageous in deriving these MC moves due to a remarkable form invariance regardless of the independent shape variables chosen. In fact, this form of the $N P \gamma T$ ensemble with length scales inside the integrals is very similar to the $N V \gamma T$ ensemble previously proposed in our group. ${ }^{3}$ It would be interesting to explore the extension of this formalism to fully flexible simulation cells where previous workers have noted the emergence of a factor $V^{-2}$ when transforming the standard NPT partition function, ${ }^{29,30}$ though we do not expect the full form invariance to remain when the rectilinear shape of the simulation box is altered.

We also show that straightforward implementations of $N P_{\|} P_{\perp} T$ are not formally equivalent to schemes for $N P \gamma T$ with $\gamma=0$. However, using our proposed NP $\gamma T$ formalism, we may very easily derive MC move pairs that vary $L_{\|}$and $L_{\perp}$ while maintaining a pressure $P$ and any choice of surface tension $\gamma$, in analogy with approaches for $N P_{\|} P_{\perp} T$.

Importantly, for all practical purposes, all MC move sets explored in this paper sample quantitatively similar average shapes and shape fluctuations. Therefore, using anisotropic pressure coupling in molecular dynamics simulations is a reasonable route to sampling the NP $\mathrm{NT}$ ensemble with $\gamma=0$, provided that the barostat samples the correct ensemble with either a factor of $1 / V$ inside the integral or a factor of $1 / V_{0}$ outside the integral. This caveat is important as not all barostats sample the correct ensemble. ${ }^{31}$ Of course, while the differences from the correct sampling scheme are too small to have any practical consequences for the systems we have used for comparison, this is not a guarantee that quantitative similarity holds for every system. Therefore, we urge researchers to use the forms that are guaranteed to sample the ensemble correctly, in particular as they do not increase the computational costs.

\section{APPENDIX: DERIVATION OF THE NPYT PARTITION FUNCTION}

In the following appendix, we connect the form of $Z_{N P \gamma T}$ used in the paper based on Attard's formulation with the "shell particle" approach of Corti and co-workers. The latter approach was designed to ensure that for each volume $V$ only states with particles occupying the full extent of the volume are counted in the partition function; otherwise volume states are overcounted in the NPT partition function. ${ }^{18-20}$ Corti achieves this in Monte Carlo simulations by requiring that one of the particles, a "shell" particle, resides in the furthest extent of the volume, $\mathrm{d} V$. This constraint leads to a new NVT partition function $Z^{*}(N, V, T)$ in the integrand of the NPT partition function, including this constraint on the "shell particle".

$$
Z_{N P T}=N \int \mathrm{d} V \mathrm{e}^{-\beta P V} Z^{*}(N, V, T)
$$

The argument for the NPT partition function overcounting states may be best understood from the schematic in Figure 8.

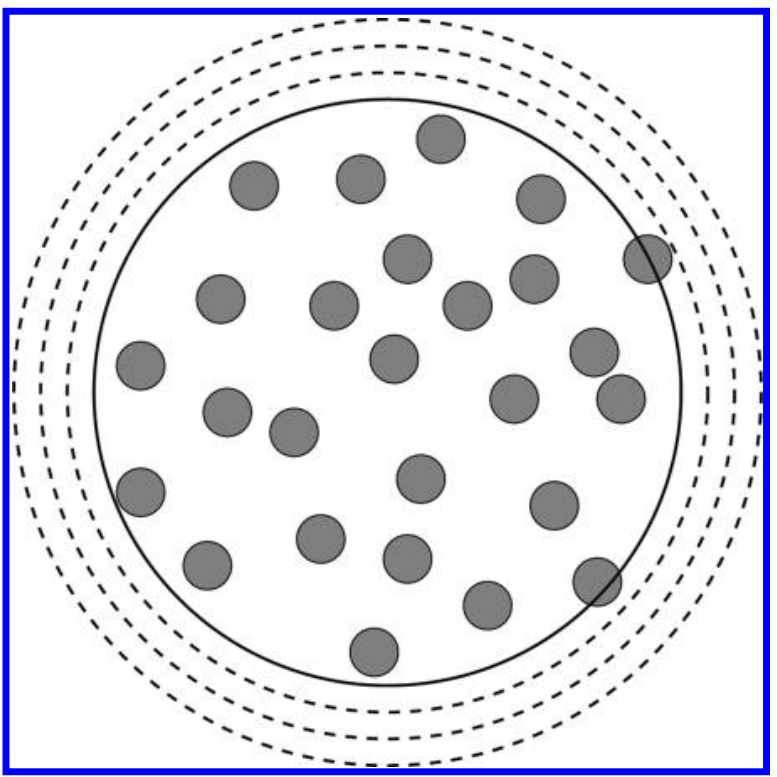

Figure 8. Argument for the NPT partition function overcounting states. The displayed configuration of particles would be counted in a canonical partition function for each of the displayed volumes (solid line and dashed lines), thus overcounting this state. However, if a particle is constrained to lie at the boundary of the volume, only the partition function for the smallest volume containing that configuration indicated with the solid line would count this configuration. Figure inspired by the diagram and discussion in ref 19 .

Unless one particle is constrained to exist at the volume boundary, the configuration shown would be counted in the partition function for the smallest volume including all the particles as well as all the partition functions for larger volumes.

However, when constructing the partition function for a system simulated with periodic boundary conditions, we find the overcounting of states to be far less serious. Since particle positions are periodically replicated, setting the system size as the position of one particle simultaneously alters the surrounding pressure bath of periodically replicated images as seen in Figure 9, and therefore $Z(N, V, T)$ determined via PBC does not implicitly include configurations from smaller volumes.

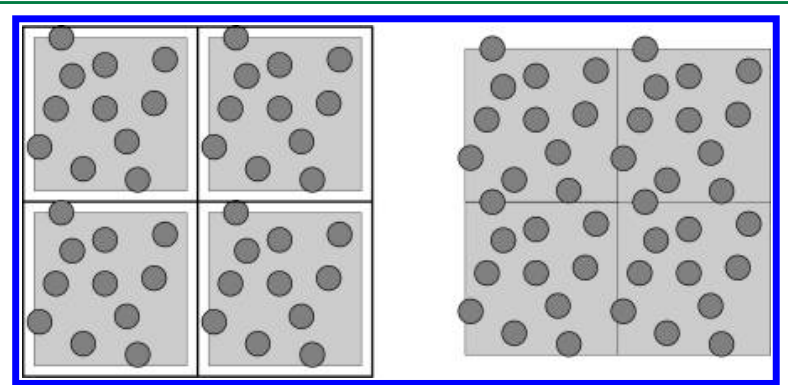

Figure 9. Demonstration that when PBCs are invoked, the canonical partition function at a larger volume does not contain configurations present in partition functions for smaller volumes. For a given volume, a configuration that occupies a slightly smaller volume within each of the periodic replicas (left) is quite different from that configuration confined to a periodically replicated simulation box of the smaller volume.

This suggests that state overcounting as described in the various works of Corti and co-workers might not be an endemic issue when periodic boundary conditions are employed. In ref 20, Corti emphasizes that the volume scale entering in the NPT 
partition function depends on the form of the boundary between the system and the pressure bath. When this boundary is determined simply by particle positions in the system, the shell particle formulation is the correct formulation of the NPT ensemble. In contrast, when the boundary is a physical object described by the system Hamiltonian with its own momentum, Corti argues that the volume scale is a constant, as in the traditional NPT formulation. We argue that NPT simulation of a periodically replicated system is a boundary type distinct from each of the two above. Particle position alone is not sufficient to determine the boundary as the origin of the periodic replication may be continually shifted. Yet, the boundary is not a physical object distinct from the particles within the system, as moving the boundary changes the interactions between particles within the simulation box and the periodically replicated images of those particles.

In the main body of this paper, we have circumvented these subtle considerations by instead presenting the arguments of Attard ${ }^{11}$ based on information theory, leading to a volume scale of $1 / V$ and a partition function of the form

$$
Z_{N P T}=N \int \frac{\mathrm{d} V}{V} \mathrm{e}^{-\beta P V} Z(N, V, T)
$$

Han and Son have previously shown that for uniform, homogeneous systems, the approach of Attard is equivalent to that of Corti. ${ }^{21}$

Here, we build on the proofs contained within refs 21 and 28 to demonstrate that in fact the approach of Attard is equivalent to that of Corti and co-workers for general nonuniform systems containing periodic boundary conditions. As we shall show, when basic features of systems simulated with PBC are considered, the state-counting encapsulated by the shell particle formalism reduces to simply a density of volume states inversely proportional to the volume.

First, we briefly review the equivalence for a uniform, homogeneous, single-component system, contained within a cube of side length $L .^{21,28}$ We require that particle 1 lies in the box boundary in the $x$ direction:

$$
\begin{aligned}
Z_{N P T}= & \frac{3 N}{\Lambda^{3 N} N !} \int \mathrm{d} L \mathrm{e}^{-\beta P L^{3}} \int_{A} \mathrm{~d} y_{1} \mathrm{~d} z_{1} \\
& \int_{V} \mathrm{~d} \mathbf{r}_{2} \int_{V} \mathrm{~d} \mathbf{r}_{3} \cdots \int_{V} \mathrm{~d} \mathbf{r}_{N} \mathrm{e}^{-\beta U_{N}}
\end{aligned}
$$

The factor of 3 arises from the fact that the $x, y$, and $z$ directions may all be equivalently chosen to set the cube side $L$. The partition function from $\int_{V} \mathrm{dr}_{2}$ forward may be identified with the particle density profile via the definition:

$$
\begin{aligned}
\rho(\mathbf{r}) & =\left\langle\sum_{i=1}^{N} \delta\left(\mathbf{r}-\mathbf{r}_{i}\right)\right\rangle \\
& =\frac{N \int \mathrm{d} \mathbf{r}_{2} \ldots \int \mathrm{d} \mathbf{r}_{N} \mathrm{e}^{-\beta U_{N \mid}} \mathbf{r}_{1}}{\int \mathrm{d} \mathbf{r}_{1} \int \mathrm{d} \mathbf{r}_{2} \ldots \int \mathrm{d} \mathbf{r}_{N} \mathrm{e}^{-\beta U_{N}}}
\end{aligned}
$$

Since $\rho(\mathbf{r})=N / V$ for a uniform system, we find exactly

$$
Z_{N P T}=3 N \int \frac{\mathrm{d} L}{L} \mathrm{e}^{-\beta P L^{3}} Z\left(N, L^{3}, T\right)
$$

with a weighting of $1 / L$ for the integrand $L$ as indicated by Attard. This equivalence between shell particle MC moves and typical volume MC moves with an acceptance ratio derived from the partition function above was also demonstrated in simulation by Corti. ${ }^{28}$ However, this equivalence would appear to break down for a nonuniform system as the density profile is no longer a constant.

However, homogeneity is not a necessary condition to yield the $1 / L$ factor in the partition function. In fact, only periodic boundary conditions (PBC) are needed. In order to derive this, we make the use of PBC explicit in the partition function. We first define $L \sigma_{o}$ as the location of the origin within the simulation box. Thus, $\sigma_{o}=0.5$ corresponds to the center of the box being the origin $x=0$. Setting $\sigma_{o}=0$ places $x=0$ at the left side of the box, and $\sigma_{o}=1$ places $x=0$ at the right side of the box. Several of these choices are shown in Figure 10 for two

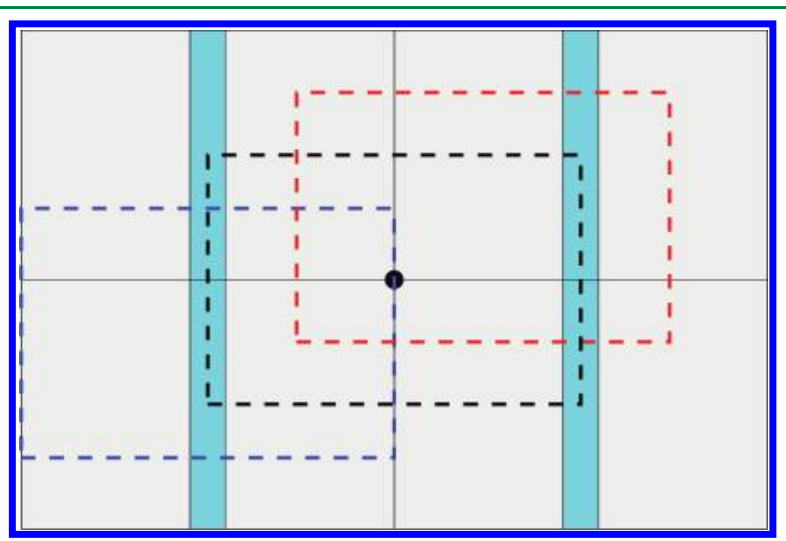

Figure 10. Schematic of four periodic replicas of the lipid bilayer system with the bilayer in cyan and the solvent in light gray. The black circle is the origin. The four boxes shown with light solid lines correspond to choices of simulation box with $\left(\sigma_{o}^{\|}, \sigma_{o}^{\perp}\right)$ set to $(0,0),(1,0),(1,1)$, and $(0,1)$ (clockwise from top right). The dashed boxes represent three additional choices of the location of the origin that may simultaneously define the main simulation box. The black dashed box corresponds to $\sigma_{o}^{\|}=0.5$ and $\sigma_{o}^{\perp}=0.5$. The red dashed box corresponds to $\sigma_{o}^{\|}=0.25$ and $\sigma_{o}^{\perp}=0.25$. The blue dashed box has $\sigma_{o}^{\|}=0.75$ and $\sigma_{o}{ }^{\perp}=1.0$. Each choice of the main simulation box is completely valid and equivalent, and using PBC implicitly includes all possible choices of origin in the simulation.

dimensions using the bilayer simulation box. When PBC simulations are conducted, all choices for $\sigma_{o}$ are included implicitly, and this fact is crucial in proving the equivalence of the shell particle approach and Attard's inverse length density of states.

We first define $Z_{N P T}\left(\sigma_{o}\right)$ with a specific choice of $\sigma_{o}$ as

$$
\begin{gathered}
Z_{N P T}\left(\sigma_{o}\right)=\frac{3 N}{\Lambda^{3 N} N !} \int_{0}^{\infty} \mathrm{d} L \mathrm{e}^{-\beta P L^{3}} \int \mathrm{d} x_{1} \delta\left(x_{1}-L\left(1-\sigma_{o}\right)\right) \\
\times \int_{A} \mathrm{~d} y_{1} \mathrm{~d} z_{1} \int_{V} \mathrm{~d} \mathbf{r}_{2} \int_{V} \mathrm{~d} \mathbf{r}_{3} \cdots \int_{V} \mathrm{~d} r_{N} \mathrm{e}^{-\beta U_{N}} \\
=3 \int_{0}^{\infty} \mathrm{d} L \mathrm{e}^{-\beta P L^{3}} \int \mathrm{d} x_{1} \delta\left(x_{1}-L\left(1-\sigma_{o}\right)\right) \\
\quad \times \int_{A} \mathrm{~d} y_{1} \mathrm{~d} z_{1} \rho\left(x_{1}, y_{1}, z_{1}\right) Z(N, V, T) \\
=3 \int_{0}^{\infty} \mathrm{d} L \mathrm{e}^{-\beta P L^{3}} \int_{A} \mathrm{~d} y_{1} \mathrm{~d} z_{1} \rho\left(L\left(1-\sigma_{o}\right), y_{1}, z_{1}\right) Z(N, V, T)
\end{gathered}
$$

The use of the delta function $\delta\left(x_{1}-L\left(1-\sigma_{o}\right)\right)$ makes the placement of particle 1 at the rightmost boundary of the simulation box explicit. However, logically, we know that the choice of the origin $\sigma_{o}$ for PBC should not impact the results of the NPT simulations at all. Furthermore, for each configuration sampled in PBC simulations, all choices of $\sigma_{o}$ 
are implicitly included and equally sampled. We therefore collect all choices of $\sigma_{o}$ to yield the full $Z_{N P T}$ for periodic boundary conditions:

$$
\begin{aligned}
& Z_{N P T}=\int_{0}^{1} \mathrm{~d} \sigma_{o} Z_{N P T}\left(\sigma_{o}\right) \\
& =3 \int_{0}^{\infty} \mathrm{d} L \mathrm{e}^{-\beta P L^{3}} Z(N, V, T) \int_{0}^{1} \mathrm{~d} \sigma_{o} \\
& \times \int_{A} \mathrm{~d} y_{1} \mathrm{~d} z_{1} \rho\left(L\left(1-\sigma_{o}\right), y_{1}, z_{1}\right) \\
& =3 \int_{0}^{\infty} \mathrm{d} L \mathrm{e}^{-\beta P L^{3}} Z(N, V, T) \frac{1}{L} \int_{0}^{L} \mathrm{~d} \xi \\
& \times \int_{A} \mathrm{~d} y_{1} \mathrm{~d} z_{1} \rho\left(\xi, y_{1}, z_{1}\right) \\
& =3 N \int_{0}^{\infty} \frac{\mathrm{d} L}{L} \mathrm{e}^{-\beta P L^{3}} Z(N, V, T)
\end{aligned}
$$

In the final equality, we have used the fact that integrating $\rho(\mathbf{r})$ over the whole volume yields $N$. This partition function is identical to that proposed by Attard when transforming the NPT partition function to that of a cube.

Strictly speaking, the derivation above is inconsistent because we postulate a nonuniform system yet couple all three directions together as a cubic simulation box. A more complete derivation for the bilayer system involves decoupling the box dimensions in the directions tangential and normal to the bilayer surface.

Here, we sketch through a derivation allowing for this decoupling. In such a case, two particles must be chosen to mark the extent of the system in the $x$ and $z$ directions, and the choice of the origin in three dimensions is indicated by $\sigma_{o}^{\|}$, and $\sigma_{o}^{\perp}$. This yields, for an explicit choice of $\sigma_{o}^{\|}$and $\sigma_{o}^{\perp}$, including the prefactor of 2 , indicating that the $y$ direction is equivalent to the $x$ direction:

$$
\begin{aligned}
& Z_{N P \gamma T}\left(\sigma_{o}^{\|}, \sigma_{o}^{\perp}\right)=\frac{2 N(N-1)}{\Lambda^{3 N} N !} \int_{0}^{\infty} \mathrm{d} L_{\|} \int_{0}^{\infty} \mathrm{d} L_{\perp} \mathrm{e}^{-\beta P V} \mathrm{e}^{\beta \gamma A} \\
& \quad \times \int \mathrm{d} x_{1} \delta\left(x_{1}-L_{\|}\left(1-\sigma_{o}^{\| \prime}\right)\right) \int_{L_{\|} L_{\perp}} \mathrm{d} y_{1} \mathrm{~d} z_{1} \\
& \quad \times \int \mathrm{d} z_{2} \delta\left(z_{2}-L_{\perp}\left(1-\sigma_{o}^{\perp}\right)\right) \int_{L_{\|} L_{\|}} \mathrm{d} x_{2} \mathrm{~d} y_{2} \\
& \quad \times \int_{V} \mathrm{~d} \mathbf{r}_{4} \int_{V} \mathrm{~d} \mathbf{r}_{5} \ldots \int_{V} \mathrm{~d}_{N} \mathrm{e}^{-\beta U_{N}} \\
& =2 \int_{0}^{\infty} \mathrm{d} L_{\|} \int_{0}^{\infty} \mathrm{d} L_{\perp} \mathrm{e}^{-\beta P V} \mathrm{e}^{\beta \gamma A} Z(N, V, A, T) \\
& \quad \times \int_{L_{\|} L_{\perp}} \mathrm{d} y_{1} \mathrm{~d} z_{1} \int_{L_{\|} L_{\|}} \mathrm{d} x_{2} \mathrm{~d} y_{2} \\
& \quad \times \rho^{(2)}\left(L_{\|}\left(1-\sigma_{o}^{\|}\right), y_{1}, z_{1} ; x_{2}, y_{2}, L_{\perp}\left(1-\sigma_{o}^{\perp}\right)\right)
\end{aligned}
$$

In the final line, $\rho^{(2)}\left(\mathbf{r}_{1} ; \mathbf{r}_{2}\right)$ is the two-particle density function. Following the same procedure as conducted for the cubic nonuniform case, we integrate $Z_{N P \gamma T}\left(\sigma_{o}^{\|}, \sigma_{o}^{\perp}\right)$ over all choices of origin in the periodic boundary conditions to yield the true $Z_{N P \gamma T}$ :

$$
Z_{N P \gamma T}=\int_{0}^{1} \mathrm{~d} \sigma_{o}^{\|} \int_{0}^{1} \mathrm{~d} \sigma_{o}^{\perp} Z_{N P T}\left(\sigma_{o}^{\|}, \sigma_{o}^{\perp}\right)
$$

$$
\begin{aligned}
= & 2 \int_{0}^{\infty} \mathrm{d} L_{\|} \int_{0}^{\infty} \mathrm{d} L_{\perp} \mathrm{e}^{-\beta P V} \mathrm{e}^{\beta \gamma A} Z(N, V, A, T) \\
& \times \int_{0}^{1} \mathrm{~d} \sigma_{o}^{\|} \int_{L_{\|}} \mathrm{d} y_{1} \int_{L_{\perp}} \mathrm{d} z_{1} \int_{L_{\|}} \mathrm{d} x_{2} \int_{L_{\|}} \mathrm{d} y_{2} \\
& \times \int_{0}^{1} \mathrm{~d} \sigma_{o}^{\perp} \times \rho^{(2)}\left(L_{\|}\left(1-\sigma_{o}^{\|}\right), y_{1}, z_{1} ; x_{2}, y_{2}, L_{\perp}\left(1-\sigma_{o}^{\perp}\right)\right) \\
= & \int_{0}^{\infty} \frac{\mathrm{d} L_{\|}}{L_{\|}} \int_{0}^{\infty} \frac{\mathrm{d} L_{\perp}}{L_{\perp}} \mathrm{e}^{-\beta P V} \mathrm{e}^{\beta \gamma A} Z(N, V, A, T) \\
& \times \int_{0}^{L_{\|}} \mathrm{d} \xi^{\|} \int_{0}^{L_{\|}} \mathrm{d} y_{1} \int_{0}^{L_{\perp}} \mathrm{d} z_{1} \int_{0}^{L_{\|}} \mathrm{d} x_{2} \int_{0}^{L_{\|}} \mathrm{d} y_{2} \\
& \times \int_{0}^{L_{\perp}} \mathrm{d} \xi^{\perp} \times \rho^{(2)}\left(\xi^{\| \prime}, y_{1}, z_{1} ; x_{2}, y_{2}, \xi^{\perp}\right) \\
= & 2 N(N-1) \int_{0}^{\infty} \frac{\mathrm{d} L_{\|}}{L_{\|}} \\
& \times \int_{0}^{\infty} \frac{\mathrm{d} L_{\perp}}{L_{\perp}} \mathrm{e}^{-\beta P V} \mathrm{e}^{\beta \gamma A} Z(N, V, A, T)
\end{aligned}
$$

where the final equality follows from integrating the twoparticle density function over all space for all coordinates.

This partition function is nearly identical to that given in the body of the paper in eq 21 based on an analogy with Attard's NPT partition function. The sole difference is the prefactor of $N(N-1)$ which has no impact on Monte Carlo acceptance ratios. Alternately, we could stipulate three distinct box boundaries set by three different particles and include the requirement that $L_{x}=L_{y}$ through the dimensionless delta function $\delta\left(1-L_{x} / L_{y}\right)$ or we could derive this partition function for a mixture of different particle types. These derivations would lead to factors different from $N(N-1)$, but the content of the partition function relevant for deriving $\mathrm{MC}$ moves would remain identical.

\section{ASSOCIATED CONTENT}

\section{S Supporting Information}

Plots demonstrating the histograms and error bars collected for all shape variables when $N_{\text {lip }}=256$ and $\gamma=0.0$ or $\gamma=2.0$. This material is available free of charge via the Internet at http:// pubs.acs.org/.

\section{AUTHOR INFORMATION}

\section{Corresponding Author}

*E-mail: jrodgers78@gmail.com; berend-smit@berkeley.edu.

\section{Notes}

The authors declare no competing financial interest.

\section{ACKNOWLEDGMENTS}

This work was supported by the Chemical Sciences, Geosciences and Biosciences Division, Office of Basic Energy Sciences, Office of Science, U.S. Department of Energy, FWP number SISGRKN.

\section{REFERENCES}

(1) Deserno, M. Macromol. Rapid Commun. 2009, 30, 752-771.

(2) Bennun, S. V.; Hoopes, M. I.; Xing, C.; Faller, R. Chem. Phys. Lipids 2009, 159, 59-66.

(3) Venturoli, M.; Sperotto, M. M.; Kranenburg, M.; Smit, B. Phys. Rep. 2006, 437, 1-54.

(4) Lyubartsev, A. P.; Rabinovich, A. L. Soft Matter 2011, 7, 25-39.

(5) Kranenburg, M.; Vlaar, M.; Smit, B. Biophys. J. 2004, 87, 15961605.

(6) Zhang, Y. H.; Feller, S. E.; Brooks, B. R.; Pastor, R. W. J. Chem. Phys. 1995, 103, 10252-10266. 
(7) Risselada, H. J.; Marrink, S. J. Proc. Natl. Acad. Sci. U.S.A. 2008,

105, 17367-17372.

(8) Venturoli, M.; Smit, B. PhysChemComm 1999, 2, 45-49.

(9) Rodgers, J. M.; Webb, M.; Smit, B. J. Chem. Phys. 2010, 132, 064107.

(10) Rodgers, J. M.; Sørensen, J.; de Meyer, F. J.-M.; Smit, B. J. Phys. Chem. B 2011, DOI: 10.1021/jp207837v.

(11) Attard, P. J. Chem. Phys. 1995, 103, 9884-9885.

(12) Frenkel, D.; Smit, B. Understanding Molecular Simulation: From Algorithms to Applications, 2nd ed.; Academic Press: New York, 2002; p 118.

(13) Crisp, D. J. Surface Chemistry: Papers presented for a Discussion at a joint meeting of the Societe de Chimie Physique and the Faraday Society held at Bordeaux from 5 to 9 October 1947 in honour of Professor Hentri Devaux published as a Special Supplement to Research a Journal of Science and its Applications; Interscience Publishers, Inc.: New York, 1949; pp 17-35.

(14) Defay, R.; Prigogine, I.; Bellemans, A. Surface Tension and Adsorption; Wiley: New York, 1966; Chapter VI.6, pp 77-78.

(15) Jähnig, F. Biophys. J. 1996, 71, 1348-1349.

(16) Kwok, R.; Evans, E. Biophys. J. 1981, 35, 637-652.

(17) Feller, S.; Pastor, R. J. Chem. Phys. 1999, 111, 1281-1287.

(18) Koper, G.; Reiss, H. J. Phys. Chem. 1996, 100, 422-432.

(19) Corti, D.; Soto-Campos, G. J. Chem. Phys. 1998, 108, 79597966.

(20) Corti, D. Phys. Rev. E 2001, 64, 016128.

(21) Han, K.; Son, H. J. Chem. Phys. 2001, 115, 7793-7794.

(22) Jaynes, E. T. IEEE Trans. Syst. Sci. Cybern. 1968, 4, 227-241.

(23) Frenkel, D.; Smit, B. Understanding Molecular Simulation: From Algorithms to Applications, 2nd ed.; Academic Press: New York, 2002; p 112.

(24) Frenkel, D.; Smit, B. Understanding Molecular Simulation: From Algorithms to Applications, 2nd ed.; Academic Press: New York, 2002; p 119.

(25) Kranenburg, M.; Venturoli, M.; Smit, B. Phys. Rev. E 2003, 67.

(26) de Meyer, F. J.-M.; Benjamini, A.; Rodgers, J. M.; Misteli, Y.; Smit, B. J. Phys. Chem. B 2010, 114, 10451-10461.

(27) Español, P.; Warren, P. Europhys. Lett. 1995, 30, 191-196.

(28) Corti, D. Mol. Phys. 2002, 100, 1887-1904.

(29) Martyna, G. J.; Tobias, D. J.; Klein, M. L. J. Chem. Phys. 1994, 101, 4177-4189.

(30) Yu, T.-Q.; Alejandre, J.; Lopez-Rendon, R.; Martyna, G. J.; Tuckerman, M. E. Chem. Phys. 2010, 370, 294-305.

(31) Tuckerman, M.; Liu, Y.; Ciccotti, G.; Martyna, G. J. Chem. Phys. 2001, 115, 1678-1702. 